\title{
Logarithmic temperature profiles of turbulent Rayleigh-Bénard convection in the classical and ultimate state for a Prandtl number of 0.8
}

\author{
Guenter Ahlers ${ }^{1,2, \dagger, \ddagger}$, Eberhard Bodenschatz ${ }^{2,3,4} \uparrow$ and Xiaozhou $\mathrm{He}^{2,}$ \\ ${ }^{1}$ Department of Physics, University of California, Santa Barbara, CA 93106, USA \\ ${ }^{2}$ Max Planck Institute for Dynamics and Self-Organization (MPIDS), 37077 Göttingen, Germany \\ ${ }^{3}$ Institute for Nonlinear Dynamics, University of Göttingen, 37077 Göttingen, Germany \\ ${ }^{4}$ Laboratory of Atomic and Solid-State Physics, and Sibley School of Mechanical \\ and Aerospace Engineering, Cornell University, Ithaca, NY 14853, USA
}

(Received 4 April 2014; revised 14 July 2014; accepted 13 September 2014; first published online 9 October 2014)

We report on experimental determinations of the temperature field in the interior (bulk) of turbulent Rayleigh-Bénard convection for a cylindrical sample with an aspect ratio (diameter $D$ over height $L$ ) equal to 0.50 , in both the classical and the ultimate state. The measurements are for Rayleigh numbers $R a$ from $6 \times 10^{11}$ to $10^{13}$ in the classical and $7 \times 10^{14}$ to $1.1 \times 10^{15}$ (our maximum accessible $R a$ ) in the ultimate state. The Prandtl number was close to 0.8. Although to lowest order the bulk is often assumed to be isothermal in the time average, we found a 'logarithmic layer' (as reported briefly by Ahlers et al., Phys. Rev. Lett., vol. 109, 2012, 114501) in which the reduced temperature $\Theta=\left[\langle T(z)\rangle-T_{m}\right] / \Delta T$ (with $T_{m}$ the mean temperature, $\Delta T$ the applied temperature difference and $\langle\cdots\rangle$ a time average) varies as $A \ln (z / L)+B$ or $A^{\prime} \ln (1-z / L)+B^{\prime}$ with the distance $z$ from the bottom plate of the sample. In the classical state, the amplitudes $-A$ and $A^{\prime}$ are equal within our resolution, while in the ultimate state there is a small difference, with $-A / A^{\prime} \simeq 0.95$. For the classical state, the width of the log layer is approximately $0.1 L$, the same near the top and the bottom plate as expected for a system with reflection symmetry about its horizontal midplane. For the ultimate state, the log-layer width is larger, extending through most of the sample, and slightly asymmetric about the midplane. Both amplitudes $A$ and $A^{\prime}$ vary with radial position $r$, and this variation can be described well by $A=A_{0}[(R-r) / R]^{-0.65}$, where $R$ is the radius of the sample. In the classical state, these results are in good agreement with direct numerical simulations (DNS) for $R a=2 \times 10^{12}$; in the ultimate state there are as yet no DNS. The amplitudes $-A$ and $A^{\prime}$ varied as $R a^{-\eta}$, with $\eta \simeq 0.12$ in the classical and $\eta \simeq 0.18$ in the ultimate state. A close analogy between the temperature field in the classical state and the 'law of the wall' for the time-averaged downstream velocity in shear flow is discussed. A two-sublayer mean-field model of the temperature profile in the classical state was analysed and yielded a logarithmic $z$ dependence of $\Theta$. The $R a$ dependence of the amplitude $A$ given by the model corresponds to an exponent $\eta_{t h}=0.106$, in good agreement with the experiment. In the ultimate state the experimental result $\eta \simeq 0.18$

$\dagger$ Email address for correspondence: guenter@physics.ucsb.edu $\ddagger$ The authors belong to the International Collaboration for Turbulence Research. 
differs from the prediction $\eta_{t h} \simeq 0.043$ by Grossmann \& Lohse (Phys. Fluids, vol. 24, 2012, 125103).

Key words: Bénard convection, convection

\section{Introduction}

Turbulent convection in a fluid contained between two parallel horizontal plates and heated from below (Rayleigh-Bénard convection; RBC) has been studied intensely for many decades both theoretically and experimentally (for various reviews, see Kadanoff 2001; Ahlers 2009; Ahlers, Grossmann \& Lohse 2009b; Lohse \& Xia 2010; Chillà $\&$ Schumacher 2012). For this system, the vertical temperature change $\Delta T \equiv T_{b}-$ $T_{t}$ between the bottom temperature $T_{b}$ and the top temperature $T_{t}$ is expressed in dimensionless form by the Rayleigh number $R a$ (see (2.1) below). Most of $\Delta T$ occurs over two thin thermal boundary layers, one adjacent to each of the top and bottom plates. For typical cases where the adiabatic gradient is negligible, the bulk of the sample was long considered to have a constant temperature $T_{m} \equiv\left(T_{b}+T_{t}\right) / 2$ in the time average, although the local temperature was understood to fluctuate vigorously. This model, which is due to Malkus $(1954)$ and Priestley $(1954,1959)$ (see also the review by Spiegel 1971, §5), has served well for several decades to explain many features of this system at a semi-quantitative level.

More detailed measurements during the last two decades revealed that, while the temperature drop across the thermal boundary layers accounted for the major part of $\Delta T$ (Lui \& Xia 1998), there were significant measurable temperature variations in the bulk of the sample (see e.g. Tilgner, Belmonte \& Libchaber 1993; Brown \& Ahlers 2007). In the absence of more detailed information, these temperature profiles often were assumed to correspond to a constant temperature gradient, with the total temperature drop across the bulk only a few per cent of $\Delta T$ and dependent on $R a$, the Prandtl number $\operatorname{Pr}$ (see (2.2) below) and the radial location $\xi$ (see (2.5) below) within the sample. These bulk gradients usually were attributed to thermal dissipation due to plumes that emanate from the top and bottom boundary layers. The plumes are carried by, and due to their buoyancy in turn drive, a large-scale circulation (LSC) so that they rise or fall through the bulk; but we are not aware of a detailed theoretical explanation, based on plumes, of the bulk temperature field.

Recently it was discovered experimentally (Ahlers et al. 2012a) and confirmed by direct numerical simulation (DNS) (Stevens, Lohse \& Verzicco 2011; as reported by Ahlers et al. 2012a) that the bulk temperature profiles in a cylindrical sample of diameter $D$ and aspect ratio $\Gamma \equiv D / L=0.50$ are far richer than had been anticipated. Over the ranges of $\operatorname{Ra}, \operatorname{Pr}$ and $\xi$ studied, it turned out that the time-averaged bulk temperature

$$
\Theta(z, r) \equiv \frac{\langle T(z, r, t)\rangle-T_{m}}{\Delta T}
$$

(we denote the average over the time $t$ by $\langle\ldots\rangle$ ) varied logarithmically with the distance $z$ from the bottom or with $L-z$ from the top plate over a significant fraction of the sample height $L$, i.e.

$$
\Theta=A(r) \ln (z / L)+B(r)
$$


near the bottom and

$$
\Theta=A^{\prime}(r) \ln (1-z / L)+B^{\prime}(r)
$$

near the top plate (here $r$ is the radial distance from the vertical centreline). It was found from DNS that the amplitudes $|A|$ and $A^{\prime}$ decrease as the radial location is varied from near the sidewall towards the sample interior. In the RBC system, typical values of $|\Theta|$ are less than 0.05 because most of the temperature drop is, as mentioned, across thermal boundary layers. Nonetheless, the very existence of logarithmic profiles provokes a comparison with the 'law of the wall' for the time-averaged downstream ('streamwise') velocity in turbulent shear flows. The shear-flow law of the wall was enunciated first by Prandtl $(1925,1932)$ and von Kármán (1930) and has been studied intensely ever since (for recent reviews, see e.g. Pope 2000; Marusic et al. 2010; Smits, McKeon \& Marusic 2011). Below, in $\S 4.3$, we present a two-sublayer model for the temperature field in turbulent RBC that makes the analogy to shear flow more concrete.

Before discussing the results of the present work, we note that turbulent RBC can exist in two or more distinct states, as first discussed by Kraichnan (1962) and Spiegel (1971) and more recently by Grossmann \& Lohse (2011). For $R a$ values below a characteristic $R a^{*}$, it was expected that the system has viscous boundary layers (BLs) adjacent to the top and bottom plates, as well as the sidewalls, established by the LSC or, in the absence of an LSC, by velocity fluctuations on length scales smaller than $D$. These viscous BLs then are believed to coexist with the above-mentioned thermal BLs adjacent to the plates. We refer to this state as the 'classical' state since it is the one that has been studied experimentally for many decades; it is the state envisioned by early workers such as Malkus (1954) and Priestley (1954, 1959). For larger $R a$, it was expected that the viscous BLs become turbulent due to the shear applied to them by the LSC or the fluctuations. This state is of particular interest because it is expected to be asymptotic in the sense that it will prevail up to arbitrarily large $R a$; thus it is referred to as the 'ultimate state' (Chavanne et al. 1997) and its properties, once studied above but near $R a^{*}$, can in principle be extrapolated to the very high Rayleigh numbers relevant to various astrophysical and geophysical phenomena that are not attainable in the laboratory.

Although Kraichnan (1962) had predicted the as-yet unattainable values $R a^{*}=$ $O\left(10^{21}\right)$ or larger, a more realistic estimate (Grossmann \& Lohse 2002) based on more recent bulk Reynolds-number measurements by Qiu \& Tong (2001) and the RBC model of Grossmann \& Lohse (2000) gave $R a^{*} \simeq 10^{14}$ for a Prandtl number near unity. Experimentally it was found recently for a cylindrical sample with $\Gamma=0.50$ that the transition to the ultimate state takes place gradually over a range of $R a$, from $R a_{1}^{*} \simeq 10^{13}$ to $R a_{2}^{*} \simeq 5 \times 10^{14}$ (Ahlers et al. 2012b; He et al. 2012a,b). Earlier heat-transport measurements (Chavanne et al. 1997, 2001; Roche et al. 2010) had suggested that this transition takes place near $R a \simeq 10^{11}$; but in our view such a low value of $R a^{*}$ would be inconsistent with the shear-induced boundary-layer transition to turbulence associated with the ultimate-state transition because the shear Reynolds number is too small. There is, however, at present no alternative explanation of the transition that is clearly evident in these early heat-transport measurements but absent in other similar measurements (Wu 1991; Niemela et al. 2000) (for a recent detailed re-examination of those data, see Ahlers et al. (2012b)).

From the viewpoint of the present paper, the ultimate state is of particular interest because Grossmann \& Lohse (2011) predicted for the Boussinesq system that each of the two turbulent kinetic BLs extends all the way to the sample midplane at $z=L / 2$, thus essentially leaving no 'bulk'. The associated time-averaged temperature 
was proposed to vary logarithmically with the distance from a horizontal plate. This implies that, at least in the ideal system, there should be logarithmic temperature profiles extending away from both the top and bottom plates, and that these profiles should meet at the horizontal midplane of the sample. In a subsequent paper, Grossmann \& Lohse (2012) used a two-layer model to calculate the amplitudes of these logarithmic temperature profiles. Their interesting predictions were the original motivation for the present experimental work. Thus, we were prepared to find the Grossmann-Lohse log profiles in the ultimate state for $R a>R a_{2}^{*}$. However, to our great surprise we found very similar profiles also in the classical state for $R a<R a_{1}^{*}$.

We assume that the logarithmic temperature distributions in classical RBC have an origin not unlike the one responsible for the logarithmic profiles of the time-averaged downstream velocity in shear-flow systems such as plane Couette, Couette-Taylor or pipe flow. However, in the RBC case, the profile apparently is not dependent upon the presence of shear along the walls or plates. Rather, it seems likely to us that it is associated with the presence and spatial distribution of thermal excitations, which are usually referred to as plumes. This is demonstrated convincingly in a recent numerical study (van der Poel et al. 2013) of a two-dimensional RBC system of large aspect ratio and with periodic lateral boundary conditions where there is no sidewall shear. The authors found that there are several LSC cells, each roughly with a diameter equal to the height. While there were logarithmic vertical temperature profiles at all horizontal locations, the amplitude $A$ had a pronounced maximum at the up-flow locations. It is also at these locations that the concentration of rising plumes emitted from the thermal BLs is largest, thus showing a correlation between plume density and log-profile amplitude. Thus it seems likely that the plumes in RBC play a role similar to that of the velocity excitations ('coherent eddies') in shear flows (see e.g. Townsend 1976; Perry \& Chong 1982). However, to our knowledge a detailed theory for RBC leading from plumes to logarithmic temperature profiles is still missing. Below, in $\S 4.3 .1$, we present a 'mean-field' model consisting of two thermal sublayers. In the first the temperature profile is controlled by the molecular thermal diffusivity, and in the second it is determined by a turbulent eddy diffusivity. This model is similar to one used by Grossmann \& Lohse (2011) for the ultimate state. It leads to logarithmic temperature profiles with an estimate of the $R a$ dependence of the amplitudes $A=-A^{\prime}$ for the classical state that agrees with experiment.

In this paper we present more extensive experimental results for the logarithmic profiles of the temperature in the interior ('bulk') of a turbulent convection sample of aspect ratio 0.50 using apparatus known as the High Pressure Convection Facility (Ahlers et al. 2012a). Both the axial and the radial dependences of the coefficients of (1.2) and (1.3) were studied in the classical state over the $R a$ range from $6 \times 10^{11}$ to $10^{13}$ and in the ultimate state from $R a=7 \times 10^{14}$ to $1.1 \times 10^{15}$ (our maximum accessible $R a$ ).

After defining the parameters relevant to this work in $\S 2$, we give a brief description of the apparatus in $\S 3.1$. A detailed discussion of the temperature probes (thermistors) used in this work is provided in $\S 3.2$. There the details of the locations of these sensors are given. The results for the classical state are presented in $\S 4$. At the end of that section, in $\S 4.3$, we discuss the analogies, similarities and differences between RBC and shear flows and present a two-sublayer model for the temperature field that yields logarithmic profiles and the $R a$ dependence of their coefficients. In $\S 5$ we present results for the ultimate state. We conclude this paper with a brief summary in $\S 6$. 


\section{Relevant parameters}

For a given sample geometry, the state of the system depends on two dimensionless variables. The first is the Rayleigh number $R a$, a dimensionless form of the temperature difference $\Delta T=T_{b}-T_{t}$ between the bottom $\left(T_{b}\right)$ and the top $\left(T_{t}\right)$ plate. It is given by

$$
R a=\frac{g \alpha \Delta T L^{3}}{\kappa \nu} .
$$

Here, $g, \alpha, \kappa$ and $v$ denote the gravitational acceleration, the isobaric thermal expansion coefficient, the thermal diffusivity and the kinematic viscosity, respectively. The second is the Prandtl number

$$
\operatorname{Pr}=\frac{\nu}{\kappa} .
$$

Unless stated otherwise, all fluid properties are evaluated at the mean temperature $T_{m}=\left(T_{b}+T_{t}\right) / 2$.

The vertical heat transport from the bottom to the top plate is expressed in dimensionless form by the Nusselt number

$$
N u=\frac{\lambda_{\text {eff }}}{\lambda},
$$

where the effective conductivity

$$
\lambda_{e f f}=\frac{Q L}{A \Delta T}
$$

and where $Q$ is the heat flux and $A$ is the cross-sectional area of the cell.

For samples in the shape of right-circular cylinders like those used here, a further parameter defining the geometry is needed and is the aspect ratio $\Gamma \equiv D / L$, where $D$ is the sample diameter.

In the axial direction, we shall use $z / L$ in the lower half and $1-z / L$ in the upper half of the sample as the relevant dimensionless coordinate. For the radial position, we shall use the parameter

$$
\xi \equiv \frac{R-r}{R},
$$

where $r$ is the radial distance $(\mathrm{cm})$ from the centreline and $R=56.0 \mathrm{~cm}$ is the radius of the sample.

\section{Apparatus and procedures}

\subsection{Apparatus}

Figure 1(a) shows a schematic diagram of the apparatus (known as the High Pressure Convection Facility; HPCF). It was described in detail elsewhere (Ahlers, Funfschilling \& Bodenschatz 2009a; Ahlers et al. 2012b). In the present work we used version HPCF-IIg, a slight modification of version HPCF-IIf listed in table 1 of Ahlers et al. (2012b). As will become evident below, the two versions differed only in the number of thermistors that had been installed to probe the temperature profiles in the sample interior. Here we describe only the main features of the overall facility, and then, in $\$ 3.2$, give details about thermistors that were added for the present work. 
(a)


FIGURE 1. (a) A schematic diagram of the HPCF-II. The coordinate origin is at the centre of the bottom plate. (b) Typical values of $P r$ as a function of $R a$. The discrete levels of $\mathrm{Pr}$ are associated with corresponding discrete pressure values.

The sample cell was located inside a pressure vessel known as the 'Uboot of Göttingen', which could be pressurized with various gases. For the present work we used sulfur hexafluoride $\left(\mathrm{SF}_{6}\right)$ at an average temperature $T_{m}=21.5^{\circ} \mathrm{C}$ and at pressures that ranged from 2.0 to 17.7 bar, resulting in a Prandtl number that varied over the relatively narrow range from 0.78 to 0.86 over the $R a$ range from $10^{11}$ to $10^{15}$ as shown in figure $1(b)$. The Uboot had a volume of approximately $25 \mathrm{~m}^{3}$, and approximately $2000 \mathrm{~kg}$ of $\mathrm{SF}_{6}$ were required to fill it to the maximum pressure. The sample cell had copper plates at the top and bottom that were separated by a distance $L=224 \mathrm{~cm}$. In the lateral direction, it had a cylindrical sidewall made of a Plexiglas tube of inner diameter $D=112 \mathrm{~cm}$ and wall thickness $0.95 \mathrm{~cm}$, yielding an aspect ratio $\Gamma=0.500$. The cell was completely sealed; it had a small-diameter tube entering it through the sidewall at mid-height to permit the fluid to enter from the Uboot. During measurements, this tube was sealed by a remotely controlled valve. Various thermal shields outside the sample cell prevented parasitic heat losses or inputs.

During all measurements, the temperatures at the top and bottom plates were held constant by digital feedback loops. One such loop controlled the power going to the bottom-plate heater. The top-plate temperature was held constant by a temperaturecontrolled cooling-water circuit. For each set of conditions (pressure, $T_{m}$ and $\Delta T$ ), measurements of all thermometers and heater currents were made at time intervals of approximately $12 \mathrm{~s}$, and over a period of typically one day. The data taken during about the first eight hours were discarded to avoid the influence of transients, and the remainder were analysed. 


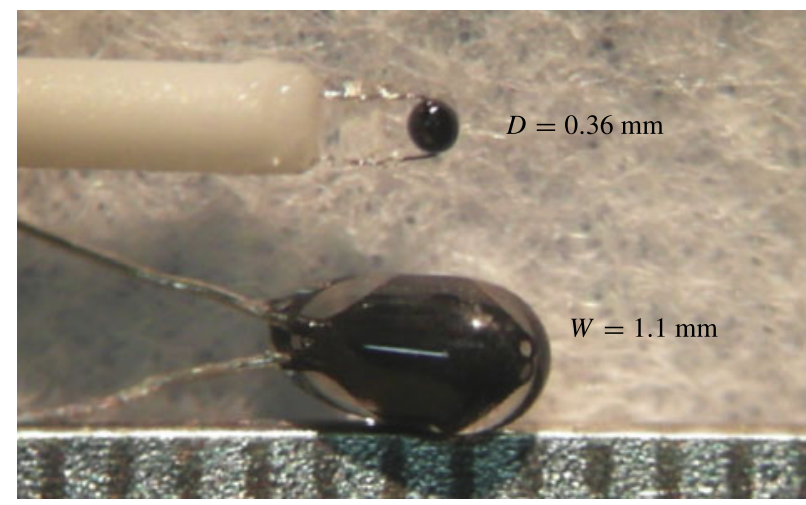

Figure 2. (Colour online) Photograph of the thermistors used in this work. The distance between the lines on the scale is $0.5 \mathrm{~mm}$.

\subsection{Thermistors used in the sample interior}

\subsubsection{Thermistor locations}

We used the two types of thermistors illustrated in figure 2. They were inserted to various depths into the sample interior. They differed in size and thus in thermal response time. While the time response is important for fluctuation measurements, for the present work of time-averaged temperature measurements both served equally well, and the two types gave equivalent results.

The larger thermistors, to be referred to as $\mathrm{T}_{1}$, were Honeywell model 112-104KAJB01. They were glass-encapsulated, and the glass capsule had an outside minor diameter of $1.1 \mathrm{~mm}$ and a length of approximately $1.8 \mathrm{~mm}$. Their platinum-alloy leads had a diameter of $0.10 \mathrm{~mm}$. They were strong enough to be passed directly through a hole in the Plexiglas sidewall of the convection cell, and to suspend the thermistor at a distance of either 0.1 or $1.0 \mathrm{~cm}$ from the inside of the sidewall. The hole in the wall was then sealed with silicone glue.

The smaller thermistors were Honeywell model 111-104HAK-H01, to be called $\mathrm{T}_{2}$. They had diameters of $0.36 \mathrm{~mm}$ and also were glass-encapsulated. Thermistors $\mathrm{T}_{2}$ had platinum-alloy leads of $0.02 \mathrm{~mm}$ diameter. The leads were passed, one each, through two $0.13 \mathrm{~mm}$ diameter holes parallel to the axis of $0.8 \mathrm{~mm}$ diameter ceramic rods (Omega ceramic thermocouple insulators type TRA-005132). The rods were placed in holes of $0.9 \mathrm{~mm}$ diameter in the Plexiglas sidewall of the convection chamber and sealed to the sidewall with silicone glue.

For the present experiment, we used the 54 thermistors listed in table 1. They were positioned in eight columns at various radial and azimuthal locations within the sample. As can be seen, version HPCF-IIf (which was used by Ahlers et al. (2012a)) contained only columns $\mathrm{V}_{0}$ and $\mathrm{V}_{1}$, both using thermistors $\mathrm{T}_{1}$. In version HPCF-IIg (which was used for the present work) eight new columns, using thermistors $\mathrm{T}_{2}$, were added at several different radial locations. For each column the vertical positions at which a thermistor was present are shown in table 2. There one sees that all columns were located in the lower half of the samples, except for $\mathrm{V}_{0}^{\prime}$ and $\mathrm{V}_{1}^{\prime}$, which were positioned in the top half so that the expected symmetry about the horizontal midplane could be investigated.

The experimental results for the logarithmic profiles reported previously by Ahlers et al. (2012a) were obtained with HPCF-IIf, which contained only the two columns 


$\begin{array}{crrccccc}\text { Column } & \begin{array}{c}d \\ (\mu \mathrm{m})\end{array} & \begin{array}{c}R-r \\ (\mathrm{~cm})\end{array} & \begin{array}{c}\xi \equiv \\ (R-r) / R\end{array} & \begin{array}{c}\theta \\ (\mathrm{rad})\end{array} & \begin{array}{c}N_{t h} \\ \text { HPCF } \\ \text { version }\end{array} & \begin{array}{c}\text { Thermistor } \\ \text { version }\end{array} \\ \mathrm{V}_{0} & 1140 & 1.0 & 0.0180 & 1.505 & 8 & \text { IIf }+\mathrm{g} & \mathrm{T}_{1} \\ \mathrm{~V}_{0}^{\prime} & 360 & 1.0 & 0.0180 & 1.505 & 4 & \text { IIg } & \mathrm{T}_{2} \\ \mathrm{~V}_{1} & 1140 & 1.0 & 0.0180 & 4.646 & 8 & \text { IIf }+\mathrm{g} & \mathrm{T}_{1} \\ \mathrm{~V}_{1}^{\prime} & 360 & 1.0 & 0.0180 & 4.646 & 4 & \text { IIg } & \mathrm{T}_{2} \\ \mathrm{~V}_{2} & 360 & 2.0 & 0.0356 & 1.095 & 5 & \text { IIg } & \mathrm{T}_{2} \\ \mathrm{~V}_{3} & 360 & 2.0 & 0.0356 & 4.236 & 5 & \text { IIg } & \mathrm{T}_{2} \\ \mathrm{~V}_{4} & 360 & 4.0 & 0.0716 & 0.963 & 5 & \text { IIg } & \mathrm{T}_{2} \\ \mathrm{~V}_{5} & 360 & 4.0 & 0.0716 & 4.105 & 5 & \text { IIg } & \mathrm{T}_{2} \\ \mathrm{~V}_{6} & 360 & 15.0 & 0.2680 & 0.518 & 5 & \text { IIg } & \mathrm{T}_{2} \\ \mathrm{~V}_{7} & 360 & 15.0 & 0.2680 & 3.660 & 5 & \text { IIg } & \mathrm{T}_{2}\end{array}$

TABLE 1. The radial and azimuthal locations of the thermistor columns. The thermistor diameter is given in the table column labelled $d$. The angle $\theta$ is the azimuthal location of the thermistor column relative to an arbitrary origin. Version HPCF-IIf was used by Ahlers et al. (2012a) and was tilted slightly, with its axis at an angle of 14 mrad relative to gravity. Version HPCF-IIg was used for the present work and was levelled relative to gravity to within $10^{-4} \mathrm{rad}$. The table column labelled $N_{\text {th }}$ gives the number of thermistors in each thermistor column.

$\mathrm{V}_{0}$ and $\mathrm{V}_{1}$, located in the lower half of the sample and each consisting of eight $\mathrm{T}_{1}$ thermistors.

\subsubsection{Thermistor calibration and method of use}

All 52 thermistors in the sample interior were calibrated inside the apparatus against the top- and bottom-plate thermistors (Ahlers et al. 2009a), which in turn had been calibrated separately against a platinum thermometer. This was done by establishing a steady state typically with an applied temperature difference of $0.8 \mathrm{~K}$ and a mean temperature $T_{m}$ close to $21.50{ }^{\circ} \mathrm{C}$. The small temperature difference was needed since the system would not reach a steady state in the absence of it, even in a time interval of a couple of days. Using parameters of the vertical logarithmic temperature profiles that were measured at various pressures and with large $\Delta T$, a small correction to the calibrations that depended on the vertical and radial location of each of the 54 thermometers was made.

Since all temperatures to be measured were close to $T_{m}$, we used the measured resistances $\Omega_{0}\left(T_{m}\right)$ and a standard value of $\Omega^{-1}(\mathrm{~d} \Omega / \mathrm{d} T)=0.0456 \mathrm{~K}^{-1}$ to calculate all temperatures.

\section{Results for the classical state}

\subsection{Results for $R a=2 \times 10^{12}$}

We chose $R a=2 \times 10^{12}$ for a detailed examination of the data because this is the largest $R a$ at which comparison with the DNS by Stevens et al. (2011) for $\operatorname{Pr}=0.7$ (see Ahlers et al. (2012a) for the results of an analysis of these data) is possible. The results at other $R a$ in the classical range $6 \times 10^{11} \lesssim R a \lesssim 10^{13}$ covered by our measurements are similar. 


$\begin{array}{rcccc}z(\mathrm{~cm}) & z / L & \mathrm{~V}_{0}, \mathrm{~V}_{1} & \mathrm{~V}_{0}^{\prime}, \mathrm{V}_{1}^{\prime} & \text { All others } \\ 4.0 & 0.0178 & \mathrm{X} & & \mathrm{X} \\ 6.1 & 0.0272 & \mathrm{X} & & \\ 8.1 & 0.0362 & \mathrm{X} & & \mathrm{X} \\ 12.1 & 0.0540 & \mathrm{X} & & \mathrm{X} \\ 16.1 & 0.0719 & \mathrm{X} & & \mathrm{X} \\ 32.2 & 0.1438 & \mathrm{X} & & \mathrm{X} \\ 64.2 & 0.2866 & \mathrm{X} & & \\ 110.5 & 0.4933 & \mathrm{X} & & \mathrm{X} \\ 188.8 & 0.8428 & & \mathrm{X} & \\ 207.9 & 0.9281 & & \mathrm{X} & \\ 217.9 & 0.9639 & & \mathrm{X} & \\ 220.0 & 0.9821 & & \end{array}$

TABLE 2. The vertical locations of the thermistors in the 10 columns defined in table 1 . The ' $\mathrm{X}$ ' for a given column indicates that that column contained a thermistor at that vertical position.

\subsubsection{Axial dependence: the logarithmic temperature profiles}

In figure $3(a, b)$ we show results for $\Theta$ from run $1209041\left(P=2.065 \mathrm{bar}, T_{m}=\right.$ $\left.21.5 \mathrm{~K}, R a=1.99 \times 10^{12}, \operatorname{Pr}=0.786\right)$ as a function of $z / L$ or $1-z / L$ at the radial position $\xi=(R-r) / R=0.018$. The open circles in panel $(a)$, to be referred to as $\Theta_{0}$, are from thermistor columns $\mathrm{V}_{0}$ and $\mathrm{V}_{0}^{\prime}$, while the open squares $\left(\Theta_{1}\right)$ are from columns $\mathrm{V}_{1}$ and $\mathrm{V}_{1}^{\prime}$. As seen from table 1, these two column pairs are located at the same radial position but at azimuthal positions $\phi$ that differ by $\pi$. Over the measuring positions from $z / L=0.018$ to 0.982 , the vertical time-averaged temperature $T(z / L)$ only changed by $13 \%$ of $\Delta T$. This indicates that the measured temperature profile is well inside the bulk, outside the thermal boundary layers. There is a small systematic difference between the temperatures at the two azimuthal locations. At the horizontal midplane, where we define $\phi \equiv \Theta(z=L / 2)$, this difference corresponds to $\phi_{0}-\phi_{1}=$ -0.0025 . With $\Delta T=9.00 \mathrm{~K}$ for this run, this indicates a temperature difference of $-0.022 \mathrm{~K}$. We attribute this difference to a dependence on azimuthal position of the time-averaged temperatures along vertical lines near the sidewall due to the preferred orientation of a weak remnant of an LSC. For all subsequent analyses, we shall use the average $\Theta \equiv\left(\Theta_{0}+\Theta_{1}\right) / 2$ of the two columns, which is shown as solid circles (red online) as a function of $z / L$ in figure $3(a, b)$.

\subsubsection{Non-Boussinesq effects and parameter estimates}

Before proceeding further, we first show that non-Oberbeck-Boussinesq (non-OB) effects do not have an undue influence on the remainder of our analysis.

For the OB case we expect that $\phi=\left(\phi_{0}+\phi_{1}\right) / 2=0$. One sees that there is a slight offset of $\Theta$ at $z / L=0.5$, corresponding to $\phi=-0.0046$ or $T_{c}-T_{m}=-0.041 \mathrm{~K}\left(T_{c}\right.$ is the temperature in the horizontal midplane of the sample, which, as we shall see below, is nearly independent of the radial position $r$ ). We regard this as indicative of small deviations of the physical system from the OB approximation (Oberbeck 1879; Boussinesq 1903), as discussed in a number of publications (see e.g. Wu \& Libchaber 1991; Zhang, Childress \& Libchaber 1997; Ahlers et al. 2006, 2007, 2008). The result for $\phi$ has the same sign as and a magnitude consistent with those for compressed ethane gas (not too close to the critical point) reported by Ahlers et al. 
(a)

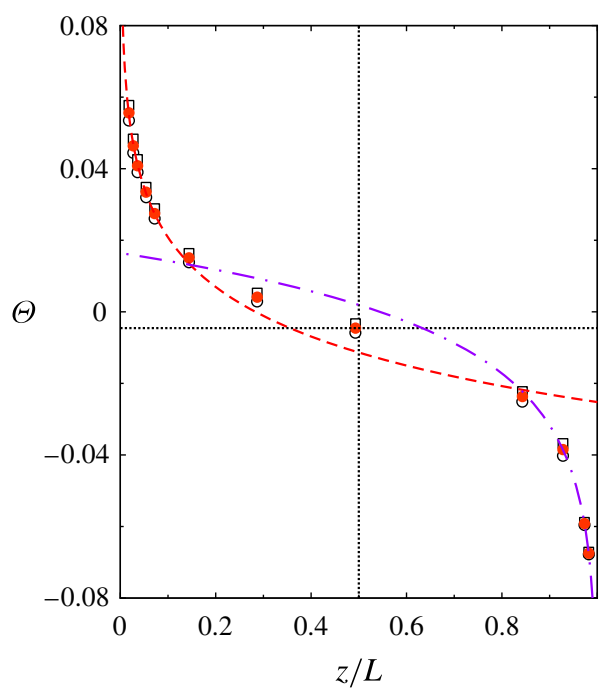

(b)

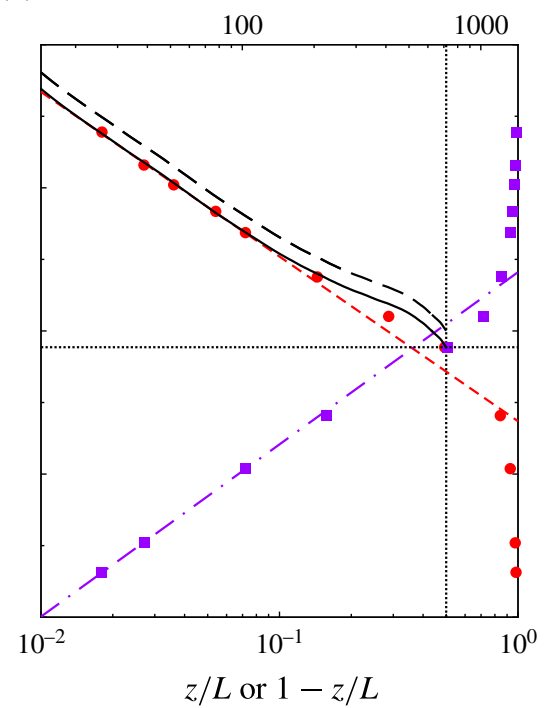

FIGURE 3. (Colour online) The dimensionless temperature $\Theta(z / L)=\left[T(z / L)-T_{m}\right] / \Delta T$ as a function of $z / L$ or $1-z / L$ for $\xi=0.0180$ from run 1209041 for $R a=1.99 \times 10^{12}$ and $\operatorname{Pr}=0.786$. (Results from run 1209051 at the same $R a$ and $\operatorname{Pr}$ were, within the resolution of the figure, virtually indistinguishable from these data.) Symbols: open circles, $\Theta_{0}$; open squares, $\Theta_{1}$; solid circles, $\Theta=\left(\Theta_{0}+\Theta_{1}\right) / 2$. (a) The data on linear scales (as a function of $z$ only); $(b)$ the data on semi-logarithmic scales. The dashed and dash-dotted lines are fits of (1.2) and (1.3) to the data for $z / L<0.08$ and $1-z / L<0.08$, respectively. They correspond to $A=-0.0200 \pm 0.0005, B=-0.0206 \pm 0.0015, A^{\prime}=0.0209$ and $B^{\prime}=$ 0.0210 . The vertical dotted line is located at $z / L=1 / 2$ and the horizontal dotted line is at $\Theta(z / L=0.5)=\phi=-0.0046$. The long-dashed line represent the DNS results of Stevens et al. (2011) for $R a=2 \times 10^{12}, \operatorname{Pr}=0.7$ and $\xi=0.0180$. The solid line is the DNS results offset by a constant increment of $\phi=-0.0046$.

(2007). Although we are not aware of a rigorous way to correct for this shift of $T_{c}$, we shall consider (when necessary) temperature profiles shifted by $\phi$, and believe that this correction is adequate in view of the small value of $\phi$.

For the Boussinesq system, we expect that the amplitudes $A$ and $A^{\prime}$ (see (1.2) and (1.3)) should have the same absolute values, i.e. $-A=A^{\prime}$. For the case shown in figure $3(b)$ we have $A=-0.0200 \pm 0.0005$ and $A^{\prime}=0.0209$ (it is not possible to determine independently a reliable uncertainty for $A^{\prime}$ and $B^{\prime}$ because the fit is based on only three data points, but we expect these uncertainties to be about the same as those for $A$ and $B$ ). One sees that, within expected uncertainties, $A=-A^{\prime}$, consistent with the OB case. Below, in $\S 4.2$, we shall examine $A / A^{\prime}$ as a function of $R a$ and see that, averaged over all $R a$ of this study, $A / A^{\prime}=-0.989 \pm 0.008$, where the uncertainty is the $67 \%$ confidence limit. Thus we find no conclusive evidence for non-OB effects on $A / A^{\prime}$. As one might have expected, this implies that the non-OB conditions lead to unequal temperature drops across the two thermal BLs adjacent to the top and bottom plates, which in turn result in a shift of $T_{c}$ relative to $T_{m}$. However, aside from this temperature shift (which is uniform throughout the bulk), the reflection symmetry about the horizontal midplane is maintained throughout the bulk of the sample. 
In order to assess more generally the possible importance of non-OB effects, we consider the dimensionless parameter $\alpha \Delta T$, which is frequently used as an empirical measure of the size of these effects. For our sample it had the value 0.033 . In the work of Urban et al. (2012), for instance, $\alpha \Delta T$ reached values as large as 0.38 . We note also that our value of $\phi$ is an order of magnitude smaller than the largest encountered by Urban et al. (2012). Thus, as discussed in more detail by He et al. (2013), there is no need for concerns such as those expressed by Urban et al. (2012) about an excessive influence of non-OB effects on our results.

\subsubsection{Comparison with direct numerical simulations}

The DNS results of Stevens et al. (2011) (see figure 3 of Ahlers et al. (2012a)) for $R a=2 \times 10^{12}, \operatorname{Pr}=0.7$ and $\xi=0.0180$ are shown as a dashed line in figure $3(b)$. These results are an azimuthal average of the computed temperature field for the $\mathrm{OB}$ system. For them $\phi=0$ and thus they are offset vertically relative to the experimental data. In order to allow a better comparison with the experiment, we shifted them by a constant increment of $\phi=-0.0046$ as found from the experiment. This yielded the solid line in figure $3(b)$, which over its range of reliability agrees superbly with the measurements. A fit of (1.2) to the DNS data over the range $0.018 \leqslant z / L \leqslant 0.072$ used in the fit to the experimental data gave $A=-0.0201$, in quantitative agreement with the measurements.

\subsubsection{Deviations from the logarithmic dependence}

Here we consider the deviations of $\Theta(z / L)$ from the fits $\Theta_{f i t}$ of (1.2) or (1.3) to the five data points with $z / L<0.08$ or the three points with $1-z / L<0.08$ when the distances from the plates become larger and approach the horizontal midplane at $z / L=1-z / L=0.5$.

From figure 3 and a close examination of the numerical data, one sees that these extrapolations reach the midplane temperature $\phi$ at $z / L=Z_{0}=0.36$ and $Z_{0}^{\prime}=1-z / L=$ 0.36 . The symmetry revealed by the finding that $Z_{0}=Z_{0}^{\prime}$, combined with the symmetry $-A=A^{\prime}$ already noted in $\S 4.1 .2$, shows that the non-OB effects of significant size are limited to the boundary layers (where they cause a uniform shift of the bulk temperature field) and do not have any other measurable influence on the bulk. Based on different measurements for a sample with $\operatorname{Pr}=4.4$ and 5.5, a similar conclusion was reached before (Brown \& Ahlers 2007). This is, of course, as expected, since temperature variations in the bulk are relatively small and thus fluid properties are nearly constant.

From (1.2) and (1.3), and our assumption that non-OB effects can be adequately compensated by a shift of the temperatures $\Theta(z / L)$ by $\phi$, one has the relations

$$
\frac{B-\phi}{A}=-\ln \left(Z_{0}\right)
$$

and

$$
\frac{B^{\prime}-\phi}{A^{\prime}}=-\ln \left(Z_{0}^{\prime}\right) .
$$

If the logarithmic profiles extended all the way to the horizontal midplane, then we would have $Z_{0}=Z_{0}^{\prime}=0.5$ and $(B-\phi) / A=\left(B^{\prime}-\phi\right) / A^{\prime}=-\ln (0.5) \simeq 0.69$. The experimental finding that $Z_{0}=Z_{0}^{\prime}<0.5$ is an indicator of the existence of an outer layer where deviations from the logarithmic profiles occur. 


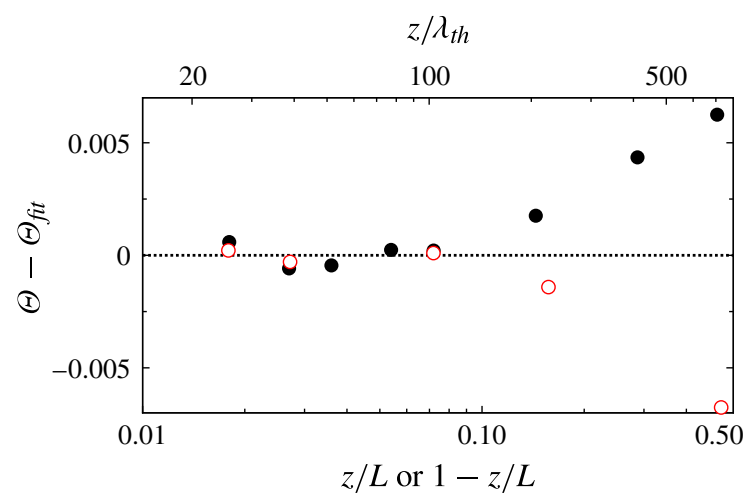

FIGURE 4. (Colour online) Deviations of the data in figure $3\left(R a=1.99 \times 10^{12}\right)$ from a fit of (1.2) to the points for $z / L<0.1$ (solid circles) or of (1.3) to the points with $1-z / L<$ 0.1 (open circles) as a function of $z / L$ or $1-z / L$ and of $z / \lambda_{t h}$ on a logarithmic scale. For this $R a$, the measurements yielded $N u=716$, giving $\lambda_{t h} / L=1 /(2 N u)=7.0 \times 10^{-4}$.

The parameters $(B-\phi) / A$ and $\left(B^{\prime}-\phi\right) / A^{\prime}$ are convenient indicators (but not a direct measure) of the widths of the logarithmic and the outer layers which can be derived directly from the parameters of the logarithmic fits to the data. Thus, below in figure $8(a)$, we show results for them as a function of $R a$. The data indicate that these widths are independent of $R a$ within the resolution of the measurements. They yield average values $(B-\phi) / A=0.967 \pm 0.023$ and $\left(B^{\prime}-\phi\right) / A^{\prime}=0.953 \pm 0.022$ (corresponding to $Z_{0}=0.380$ and $Z_{0}^{\prime}=0.386$ ), indicating that the outer layer remains symmetric about the midplane and of the same width independent of $R a$.

Next we examine more directly the deviations of $\Theta(z / L)$ from $\Theta_{f i t}$. As $z / L$ or $1-$ $z / L$ exceed 0.1 , one can see from figure 3 that deviations occur. This is more apparent in the plot of $\Theta-\Theta_{f i t}$ as a function of $z / L$ shown in figure 4 . For $z / L \lesssim 0.1$ the root-mean-square (r.m.s.) deviations are less than approximately $5 \times 10^{-4}$, i.e. $0.05 \%$ of $\Delta T \simeq 9 \mathrm{~K}$ or approximately $4 \mathrm{mK}$. For larger $z / L$ they increase, reaching values near 0.006 (or $0.6 \%$ of $\Delta T$ or $54 \mathrm{mK}$ ) at the sample midplane. The existence of a log layer and an outer layer now can be seen clearly. This is analogous to results for various shear flows (see e.g. Pope 2000); this analogy will be discussed in detail in $\S 4.3$.

\subsubsection{Radial dependence of $\Theta$}

An important aspect of $\mathrm{RBC}$ is the existence of an additional coordinate that does not exist in shear flow. In RBC the parameters of the log profiles can, and indeed do, vary in the radial direction. This was observed first in DNS (Stevens et al. 2011; see Ahlers et al. 2012a), and is illustrated in figure 5, which shows experimental data for $\Theta$ as a function of $z / L$ for four radial positions and $R a=1.99 \times 10^{12}$. One sees that the slopes of the lines fitted to the points (on a semi-log scale) decrease as the sample centreline $(\xi=(R-r) / R=1)$ is approached. At the horizontal midplane of the sample $(z / L=0.5)$, the data at all radial positions $\xi$ nearly coincide, showing that there is very little variation of the temperature in that plane.

Figure 6 shows the parameters $A$ and $B-\phi$ for two separate runs at $R a=1.99 \times$ $10^{12}$ as a function of $\xi$. The two datasets are in near-perfect agreement. Here the midplane temperature shift $\phi=\Theta(z / L=0.5)=\left(T_{m}-T_{c}\right) / \Delta T$ was subtracted from $B$ so as to compensate for the non-OB effects. One sees that the magnitudes of $A$ 


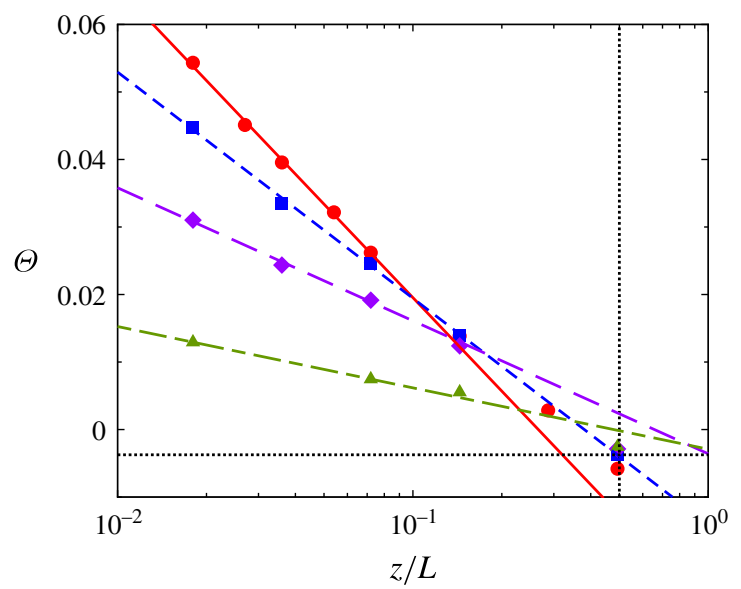

FIgURE 5. (Colour online) Results at the four radial locations $(R-r) / L$ for $\Theta(z)$ from run $1209041\left(R a=1.99 \times 10^{12}, \operatorname{Pr}=0.786\right)$ from HPCF-IIg. Near the left edge of the graph we have, from top to bottom, $\xi=(R-r) / R=0.0180,0.0356,0.0716$ and 0.2680 . Note that all four datasets have very nearly the same value at $z / L=0.5$, showing that the horizontal midplane temperature $\phi \equiv \Theta(z / L=0.5)$ is nearly independent of $r$. The averaged measured value $\langle\phi\rangle=-0.0037$ is shown as a horizontal dotted line. The vertical dotted line is at $z / L=0.5$, corresponding to the horizontal midplane.

and of $B-\phi$ decrease as the radial position decreases. This result is not surprising if one assumes that the logarithmic profile of $\Theta$ is the result of the plumes that are emitted from the thermal boundary layers adjacent to the plates and travel upwards or downwards through the sample. Plumes tend to rise or fall where there is an upor down-flow of an LSC. Thus, for a cylindrical sample of aspect ratio near unity, plumes are found in greater abundance near the sidewalls.

We note that the observed radial variation of $A$ and $B-\phi$ presumably is a characteristic of samples with small $\Gamma$, which would not prevail for a sample with $\Gamma \gg 1$. For that laterally extended case, it is expected that there will be many LSC cells (see e.g. Bailon-Cuba, Emran \& Schumacher 2010) with plumes rising or falling (and thus relatively large amplitudes of the logarithmic temperature profile) near their boundaries. These cells are expected to diffuse randomly in the horizontal plane (Hogg \& Ahlers 2013), thus yielding a more uniform $A$ and $B$ in the time average. Clearly, experiments with large- $\Gamma$ samples would be of interest.

Also shown in figure 6, as a dashed line, is the DNS result for $\operatorname{Ra}=2 \times 10^{12}, \operatorname{Pr}=$ 0.7 (Stevens et al. 2011; see Ahlers et al. 2012a). From the DNS, azimuthal temperature averages were computed in order to increase the precision of the results. For small $(R-r) / R$, the agreement with the data is excellent; it deteriorates slightly as the sample centreline at $r=0$ is approached, probably as a result of a decrease in the accuracy of the DNS because the azimuthal averaging includes fewer grid points at the smaller radii.

Recently Grossmann \& Lohse (2012) examined the logarithmic profiles in the ultimate state and for that case proposed that the amplitude should vary as

$$
|A|=\frac{A_{1}}{\sqrt{2 \xi-\xi^{2}}} .
$$



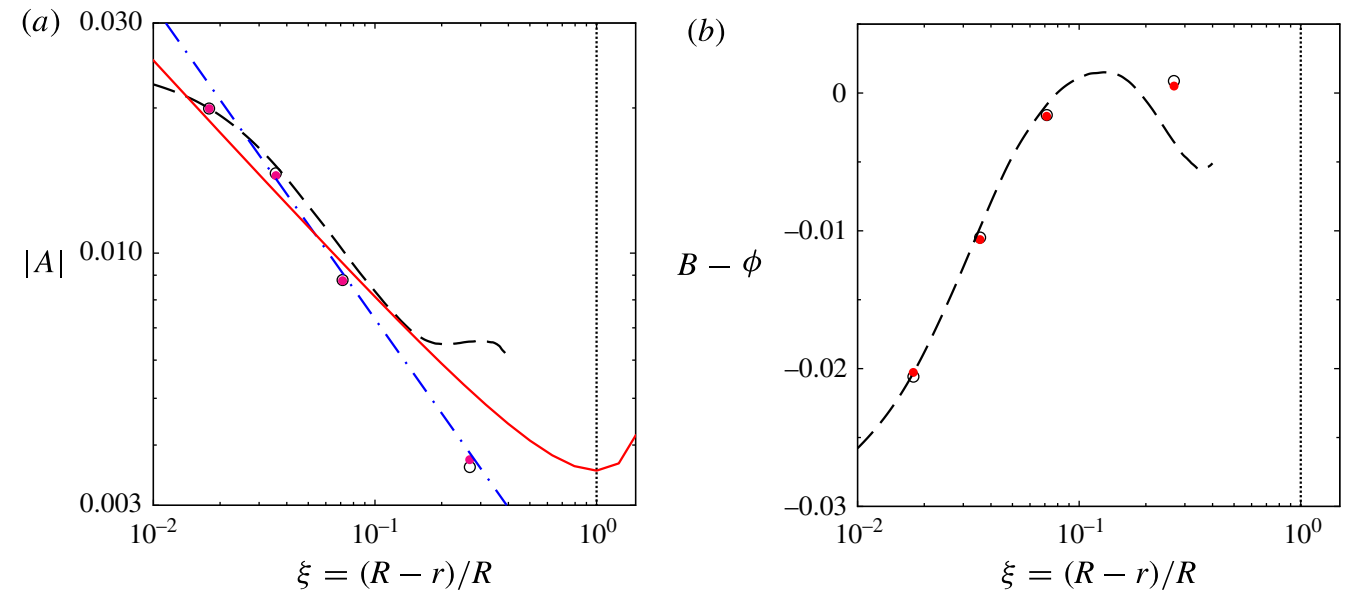

FIgURE 6. (Colour online) The parameters (a) $|A|$ and (b) $B-\phi$ of the logarithmic temperature profiles for $R a=2.0 \times 10^{12}$ and $P r \simeq 0.787$. Open circles: run 1209041. Solid circle: run 1209 051. Dashed line: DNS by Stevens et al. (2011) (see Ahlers et al. 2012a) for $R a=2 \times 10^{12}$ and $\operatorname{Pr}=0.7$. Dash-dotted line (blue online): $|A|=0.0016 \xi^{-0.65}$ with $\xi=(R-r) / R$. Solid line (red online): (4.3) with $A_{1}=0.00354$. The vertical dotted line is located at the axis of the sample.

Near the sidewall (but inside the boundary layers) one then has $|A(\xi)| \sim\left(A_{1} / \sqrt{2}\right) \xi^{-1 / 2}$, and at the vertical centreline $|A(\xi=1)|=A_{1}$. Although the prediction was not for the classical state illustrated by figure 6 , it may be relevant regardless of the mechanism involved in the generation of the logarithmic profiles because it is based on geometric arguments that assume a circular path of the LSC. Thus we compare it with the data by showing it as the solid line (red online) in the figure. Here the coefficient $A_{1}$ was adjusted to match the points at small $\xi$, which yielded $A_{1}=0.0035$. The data fall more rapidly with increasing $\xi$ and the $A_{1}$ estimate must be high. An alternative representation of the data is provided by the dot-dashed line (blue online), which is the power law $|A|=A_{0} \xi^{-0.65}$. Grossmann \& Lohse (2012) had indicated (for the ultimate state) that for samples like ours with an aspect ratio less than unity an exponent more negative than the value $-1 / 2$ indicated by (4.3) may be appropriate because the streamlines of the LSC will deviate more from a circle. The power law extrapolates to $A_{0}=0.0016$ at $\xi=1$, but this extrapolation cannot be valid either and must yield a low value because it leads to a singularity in $A(r)$ at $\xi=1$. An extrapolation that is rounded and has zero slope at the vertical centreline would yield a larger value, somewhere between 0.002 and 0.003 .

\subsection{Dependence of the parameters of the logarithmic term on $R a$}

In figure 7 we show the amplitude $A$ as a function of $R a$ on double-logarithmic scales for the four values of $\xi$. One sees that $A(\xi, R a)$ depends on $R a$ at all radial positions, and fits of the power law

$$
A=A_{0}(\xi) R a^{-\eta}
$$

to the data for $z / L<0.08$ or

$$
A^{\prime}=A_{0}^{\prime}(\xi) R a^{-\eta^{\prime}}
$$

to the data for $1-z / L<0.08$ (the lines in the figure) give similar exponent values. 


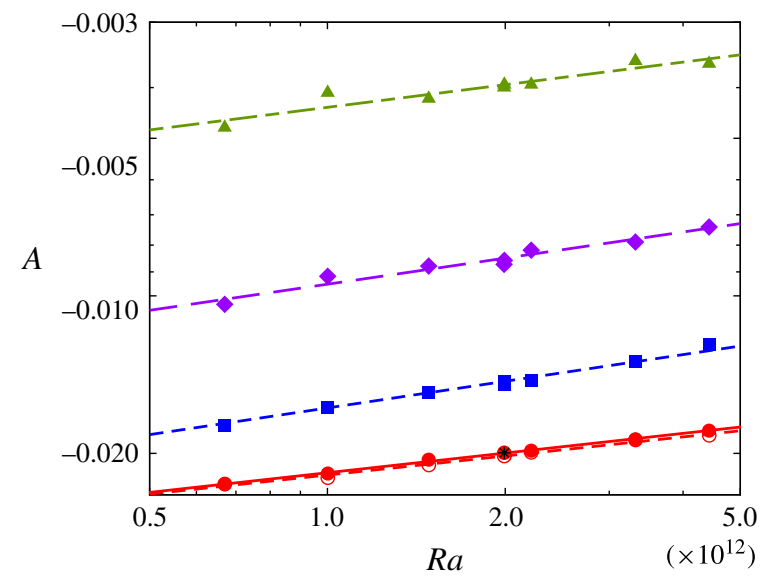

FIgURE 7. (Colour online) The amplitudes $A$ and $A^{\prime}$ of the logarithmic dependence of $\Theta$ on $z / L$ for the classical state as a function of the Rayleigh number $R a$. From bottom to top, the data are for the radial positions $\xi=0.0180,0.0356,0.0716$ and 0.268 (see tables 1 and 2). The lines are fits of (4.4) or (4.5) to the data. All data are for $A$, except that for $\xi=0.018$ the open circles (several of them hidden by the solid circles) are for $-A^{\prime}$. The black star corresponds to the data in figure 3 .

The dependences of $A$ and $A^{\prime}$ on $R a$ are not very strong, and thus accurate data are needed to determine $\eta$ and $\eta^{\prime}$ with meaningful accuracy. As can be seen from figure 5, only the data for $\xi=0.018$ are based on fits to five temperatures for $z / L<0.08$. For all other $\xi$, there were only two or three measurements and thus systematic errors arising from errors in the thermometer locations and calibrations are expected to be larger. In addition, we expect errors of the thermometer locations to increase as $\xi$ increases because the thermistors are suspended on ever increasing lengths of ceramic rods (see $\$ 3.2 .1$ ) and thus more difficult to position with high accuracy. Further, the size of the logarithmic amplitudes decreases strongly with increasing $\xi$, and thus the random errors of the measurements become more important. For these reasons we believe that only the measurements for $\xi=0.018$ (open and solid circles in figure 7) warrant a quantitative study of the $R a$ dependence of the coefficients. A fit of (4.4) and (4.5) to the data for $\xi=0.018$ yielded

$$
A_{0}=-0.686 \pm 0.06, \quad \eta=0.125 \pm 0.003
$$

and

$$
A_{0}^{\prime}=0.623 \pm 0.05, \quad \eta^{\prime}=0.121 \pm 0.003
$$

These results are consistent with $\eta=\eta^{\prime}=0.123$ with an uncertainty of 0.01 or smaller. A fit to the data with $\eta$ and $\eta^{\prime}$ fixed at 0.123 gave $A=-0.651 \pm 0.001$ and $A^{\prime}=0.659 \pm 0.002$, corresponding to the ratio $A / A^{\prime}=-0.988 \pm 0.005$. This result is consistent with the value $A / A^{\prime}=-0.989 \pm 0.008$ given in $\S 4.1 .2$ and based on an analysis of the data at a single value of $R a$. It suggests a value slightly less than unity for $-A / A^{\prime}$; but since all error estimates are $67 \%$ confidence limits, we cannot really assert that the ratio differs significantly from unity.

The dependence of $A$ on $R a$ does not have an analogue in shear flow, where the logarithmic profile of the time-averaged streamwise velocity (when scaled 


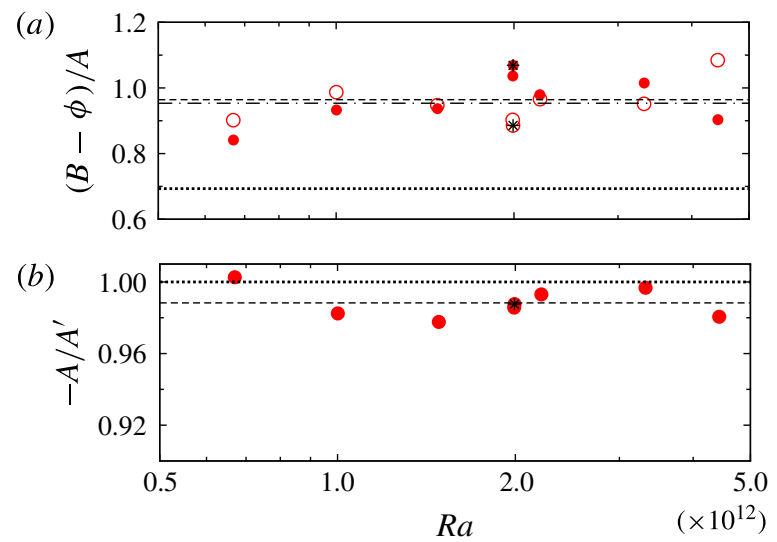

FIGURE 8. (Colour online) (a) The parameter ratio $(B-\phi) / A$ and $(b)$ the amplitude ratio $A / A^{\prime}$ of the logarithmic dependence of $\Theta$ on $z / L$ for the classical state as a function of the Rayleigh number $R a$. In panel $(a)$ the open and solid circles are for $\left(B^{\prime}-\phi\right) / A^{\prime}$ and $(B-\phi) / A$, respectively. The dotted horizontal lines correspond to the Boussinesq values $-A / A^{\prime}=1$ and $(B-\phi) / A=-\ln (0.5)=0.693$. The short-dashed and dash-dotted lines in panel $(a)$ correspond to the averaged values $\langle(B-\phi) / A\rangle=0.964$ and $\left\langle\left(B^{\prime}-\phi\right) / A^{\prime}\right\rangle=$ 0.953 , respectively. The short-dashed line in panel $(b)$ corresponds to the averaged value $\left\langle-A / A^{\prime}\right\rangle=0.989$. The black stars correspond to the data in figure 3 .

appropriately by the friction velocity) has an amplitude $1 / \kappa_{u}$, where $\kappa_{u}$ is the von Kármán constant and independent of the Reynolds number. The origin of the $R a$ dependence of $A$ will be illuminated below in $\S 4.3 .1$ where we present an analysis of a two-layer model, which yields $\eta_{t h}=0.103$, quite close to the experimental value. As discussed in $\$ 4.3 .2$, the radial dependence of $A$ does not have an analogue in the shear-flow case, as the radial dimension is unique to $\mathrm{RBC}$.

For log profiles that extend all the way to the horizontal midplane $\left(Z_{0}=Z_{0}^{\prime}=1 / 2\right)$, we expect $($ see $(4.1))(B-\phi) / A=\left(B^{\prime}-\phi\right) / A^{\prime}=-\ln (0.5) \simeq 0.69$ independent of $R a$. We show both $-A / A^{\prime}$ and $(B-\phi) / A$ in figure 8 . As already noted above, $-A / A^{\prime}$ is very close to unity and within the resolution independent of $R a$. However, $(B-$ $\phi) / A$ is larger than 0.69 . The data yield $\langle(B-\phi) / A\rangle=0.967 \pm 0.023\left(Z_{0}=0.380 \pm\right.$ $0.009)$ and $\left\langle\left(B^{\prime}-\phi\right) / A^{\prime}\right\rangle=0.953 \pm 0.022\left(Z_{0}^{\prime}=0.385 \pm 0.008\right)$. As already discussed in $\S 4.1 .4$, we attribute this to the existence of an outer layer between each log layer and the midplane. Within their resolution the data do not reveal any $R a$ dependence of the width of the log layer, and the result $Z_{0}=Z_{0}^{\prime}$ indicates that the outer layer is symmetrically distributed about the midplane.

\subsection{The 'law of the wall'}

\subsubsection{The logarithmic law for the temperature in turbulent convection}

The experimentally observed logarithmic dependence of the time-averaged temperature in turbulent $\mathrm{RBC}$ on the vertical distance from the top or bottom plate shows a strong similarity to the logarithmic dependence of the time-averaged downstream velocity in high-Reynolds-number shear flows on the distance from a wall (the 'law of the wall'; LoW) (Prandtl 1925; von Kármán 1930; Smits et al. 2011). This logarithmic dependence also shares many analogies with the temperature logarithmic law observed in fully turbulent flow in a heated pipe or along a heated 
plate (see e.g. Kader \& Yaglom 1972, and references therein). The result thus suggests that there exists a corresponding LoW for the turbulent RBC temperature field.

For fully turbulent flow in a heated pipe or along a heated plate, the temperature logarithmic law is given by

$$
\frac{T(z)-T_{w}}{T_{*}}=-\frac{1}{\kappa_{\theta}} \ln \left(\frac{z v}{u_{*}}\right)+f(P r) .
$$

Here $T_{w}$ is the time-averaged temperature at the wall $(z=0), T(z)$ is the time-averaged temperature at a distance $z$ away from the wall, and $\kappa_{\theta}$ is an empirical constant to be determined from experiment. The characteristic temperature $T_{*}$ is defined by

$$
T_{*}=\frac{J}{\rho C_{p} u_{*}},
$$

and the characteristic velocity $u_{*}$ is defined by

$$
u_{*} \equiv \sqrt{\left.v\left(\frac{\partial U}{\partial z}\right)\right|_{z=0}},
$$

where $U$ is the streamwise mean-flow velocity, $J$ is the heat flux through the wall, and $\rho$ and $C_{p}$ are the density and the specific heat of the fluid. The study of the nearwall temperature field has a long history (see e.g. Reichardt 1951). To our knowledge (4.8) was first derived from dimensional analysis by Landau \& Lifshitz (1963) (in the first Russian edition of 1944). It can also be obtained approximately by models with two or more temperature sublayers, involving for instance a diffusion sublayer with a linear conduction profile and a turbulent inner sublayer with a logarithmic profile (see Yaglom 1979; Kader 1981, and references therein).

In the study of the turbulent RBC temperature profile, a two-sublayer model was developed by Grossmann \& Lohse (2012) for the ultimate state where the boundary layers are turbulent. She et al. (2014) presented a three-sublayer model for the RBC temperature profile. For each sublayer in a model, an appropriate scaling form was proposed for the integral kernel of the time-averaged heat-transfer equation, which leads to a corresponding term in the time-averaged temperature profile after integration. Both models yield a logarithmic term in the temperature profile. Here we follow largely the procedure of Grossmann \& Lohse (2012), which involves a smaller number of parameters, and derive expressions for the amplitude $A$ and constant $B$ of the logarithmic temperature profile in classical RBC.

For an RBC sample with infinitely extended horizontal plates, it is assumed that the time-averaged temperature field near the bottom (or top) plate varies only along the vertical direction and is sheared by the flow, which has its mean speed only along the horizontal direction. The time-averaged heat-transport equation

$$
\frac{\partial}{\partial z}\left(\rho C_{p} \kappa \frac{\partial\langle T\rangle}{\partial z}-\rho C_{p}\langle w \delta T\rangle\right)=0
$$

can be written as

$$
\frac{\partial}{\partial z}\left[\rho C_{p}\left(\kappa+\kappa_{\text {turb }}\right) \frac{\partial\langle T\rangle}{\partial z}\right]=0 .
$$


Here $\langle\ldots\rangle$ indicates the time average, $w$ is the vertical component of the r.m.s. fluctuation velocity, $\delta T(t) \equiv T(t)-\langle T\rangle$ is the temperature fluctuation, and the eddy thermal diffusivity $\kappa_{\text {turb }}$ is given by

$$
\kappa_{\text {turb }} \equiv-\frac{\langle w \delta T\rangle}{\partial\langle T\rangle / \partial z} .
$$

Integrating (4.12) from the bottom plate $(z=0)$ to an arbitrary $z$, one has

$$
\rho C_{p}\left(\kappa+\kappa_{\text {turb }}\right) \frac{\partial\langle T\rangle}{\partial z}-\left.\left[\rho C_{p} \kappa \frac{\partial\langle T\rangle}{\partial z}\right]\right|_{z=0}-\left.\left[\rho C_{p} \kappa_{\text {turb }} \frac{\partial\langle T\rangle}{\partial z}\right]\right|_{z=0}=0 .
$$

The second term is the vertical heat flux $J$ at the plate, and the third term vanishes because $\kappa_{\text {turb }}=0$ at $z=0$. Rearranging and integrating on both sides, one has

$$
\int_{T_{b}}^{T(z)} \partial\langle T\rangle=\left(-\frac{J}{\rho C_{p}}\right) \int_{0}^{z} \frac{\partial z}{\kappa+\kappa_{\text {turb }}} .
$$

Here $J \equiv N u \rho C_{p} \kappa \Delta T / L$ is positive, indicating that the heat flow is in the upward direction, and the '-' sign comes from the negative mean temperature gradient. For near-wall turbulence, the characteristic velocity is $u_{*}$ (see e.g. Pope 2000). Using a similar dimensional analysis as in $\$ 54$ of Landau \& Lifshitz (1963), the eddy diffusivity close to the wall can be expressed by

$$
\kappa_{\text {turb }}=\kappa_{\theta} z u_{*} .
$$

Similarly, the kinematic eddy viscosity

$$
v_{t u r b} \equiv-\frac{\langle u w\rangle}{\partial U / \partial z}
$$

can be expressed by

$$
v_{\text {turb }}=\kappa_{u} z u_{*},
$$

where $u$ is the horizontal component of the r.m.s. fluctuation velocity and $\kappa_{u}$ is the von Kármán constant with the empirical value $\kappa_{u}=0.41$. Using the turbulent Prandtl number

$$
\operatorname{Pr}_{\text {turb }} \equiv \frac{v_{\text {turb }}}{\kappa_{\text {turb }}}
$$

one has

$$
\kappa_{\theta}=\frac{\kappa_{u}}{P r_{\text {turb }}} .
$$

In order to simplify the integration in (4.15), the RBC temperature field is assumed to consist of a marginally stable laminar (albeit time-dependent) thermal boundary layer near the plate (Zhou et al. 2010; Zhou \& Xia 2010; Stevens et al. 2012), and a turbulent interior bulk above it. In the laminar layer $\left(0 \leqslant z \lesssim z_{\theta}\right), \partial T / \partial z \gg\langle w \delta T\rangle$, leading to $\kappa \gg \kappa_{\text {turb }}$. In the turbulent bulk region $\left(z \gtrsim z_{\theta}\right)$, the fluctuation term dominates, and thus $\kappa \ll \kappa_{\text {turb }}$. The two regions merge at $z=z_{\theta}$, where $\kappa=\kappa_{\text {turb }}$. We note that Grossmann \& Lohse (2012) made the same assumption for the near-wall temperature field in the ultimate $\mathrm{RBC}$ regime, although in that case also the boundary 
layer was taken to be turbulent. The integral in (4.15) can then be split into two parts, which yields

$$
\int_{T_{b}}^{T(z)} \partial\langle T\rangle=\left(-\frac{J}{\rho C_{p}}\right)\left[\int_{z_{\theta}}^{z} \frac{\partial z}{\kappa_{\theta} z u_{*}}+\int_{0}^{z \theta} \frac{\partial z}{\kappa}\right] .
$$

With the characteristic length scale

$$
\delta_{v}=v / u_{*},
$$

(4.21) can be written as

$$
T(z)-T_{b}=\left(-\frac{J}{\rho C_{p} u_{*}}\right)\left[\frac{1}{\kappa_{\theta}} \int_{z \theta}^{z} \frac{\partial z}{z}+\frac{P r}{\delta_{v}} z_{\theta}\right],
$$

which yields

$$
\frac{T(z)-T_{b}}{T_{*}}=-\frac{1}{\kappa_{\theta}} \ln \left(\frac{z}{\delta_{v}}\right)+\frac{1}{\kappa_{\theta}} \ln \left(\frac{z_{\theta}}{\delta_{v}}\right)-\operatorname{Pr} \frac{z_{\theta}}{\delta_{v}} .
$$

This result agrees with the (4.8) when

$$
f(\operatorname{Pr})=\frac{1}{\kappa_{\theta}} \ln \left(\frac{z_{\theta}}{\delta_{v}}\right)-\operatorname{Pr} \frac{z_{\theta}}{\delta_{v}},
$$

and it is also equivalent to equation (9) of Grossmann \& Lohse (2012).

At the crossover height $z_{\theta}, \kappa=\kappa_{\theta} z_{\theta} u_{*}$, giving $z_{\theta} \equiv \kappa /\left(\kappa_{\theta} u_{*}\right)$. One can derive the relation

$$
\frac{z_{\theta}}{\delta_{v}}=\frac{P r_{t u r b}}{\operatorname{Pr}} \kappa_{u}^{-1} .
$$

The general form

$$
P r_{\text {turb }}=C_{1}+\frac{C_{2}}{P r}
$$

for the turbulent Prandtl number was given by Jischa \& Rieke (1979), with the constants $C_{1}$ and $C_{2}$ to be determined from experimental or numerical data. Numerical investigations of $P r_{\text {turb }}$ by Yu, Ozoe \& Churchill (2001) for heated channel flows have shown that $P r_{\text {turb }}$ is independent of the boundary-layer geometry over a wide range of $R e$ and $P r$, and that the two constants are $C_{1}=0.85$ and $C_{2}=0.015$ (see e.g. Churchill 2002). In turbulent RBC, we are not aware of any measurements of $C_{1}$ and $C_{2}$. We shall assume that these constants are independent of $R a$ as well as of the boundary-layer geometry when $R a$ is sufficiently large.

Given the above considerations, both $\kappa_{\theta}$ and $f(P r)$ are independent of $R a$ and constant when $P r$ is constant. Therefore (4.8) represents the $R a$-independent logarithmic profile for the time-averaged temperature near the bottom plate. A similar analysis can also be used to derive a logarithmic temperature profile near the top plate. Comparing (1.1) and (4.8), one sees that the parameter $A$ measured in the experiment is given by

$$
A=-\frac{T_{*}}{\Delta T} \frac{1}{\kappa_{\theta}}
$$


and the measured parameter $B$ is given by

$$
B=A\left[\ln \left(\frac{L}{\delta_{v}}\right)-f(P r) \kappa_{\theta}\right]+\frac{1}{2} .
$$

We note that these results are the same as equations (10) and (11) of Grossmann \& Lohse (2012) for the ultimate state. Since $\kappa_{\theta}$ depends (weakly) on $\operatorname{Pr}$ and not on $\operatorname{Ra}$, one sees that the $R a$ dependence of $A$ is determined by $T_{*} / \Delta T$.

To test this prediction, we examine the dependence of $T_{*} / \Delta T$ on $R a$. For the BLs in the classical state, the velocity and temperature derivatives can be estimated as

$$
\left.\left(\frac{\partial U}{\partial z}\right)\right|_{z=0} \sim \frac{\mathrm{V}_{e f f}}{\lambda_{u}},
$$

with $\mathrm{V}_{e f f} \equiv\left(U_{L}^{2}+\sigma_{u}^{2}\right)^{1 / 2}$ and

$$
\left.\left(\frac{\partial T}{\partial z}\right)\right|_{z=0} \sim \frac{\Delta T}{2 \lambda_{t h}} .
$$

Here $\lambda_{u}$ and $\lambda_{t h}$ are, respectively, the thicknesses of the viscous and the thermal boundary layers. Here $\mathrm{V}_{\text {eff }}$ denotes the total shear intensity on the BLs, which represents collective contributions from both the LSC mean velocity $U_{L}$ and the r.m.s. fluctuation $\sigma_{u}$ on smaller length scales. Using these estimates in (4.9) and (4.10), we have

$$
T_{*} \sim \Delta T\left(\frac{1}{2 \operatorname{Pr}}\right)\left(\frac{\lambda_{u}}{\lambda_{t h}}\right) R e_{s}^{-1 / 2}
$$

and

$$
u_{*} \sim \frac{v}{\lambda_{u}} \operatorname{Re}_{s}^{1 / 2}
$$

Here $R e_{s} \equiv \mathrm{V}_{\text {eff }} \lambda_{u} / v$ is the $\mathrm{BL}$ shear Reynolds number, which is related to the Reynolds number $R e_{\text {eff }} \equiv \mathrm{V}_{\text {eff }} L / v$ by $\operatorname{Re}_{s} \propto \operatorname{Re}_{\text {eff }}^{1 / 2}$ (Grossmann \& Lohse 2001).

To compare with the measured $\eta$ (or $\eta^{\prime}$ ), the Reynolds number $R e_{e f f}$ has to be taken from experiment or from DNS, while the BL thickness ratio $\lambda_{u} / \lambda_{t h}$ can be obtained from experiment, from DNS, or from Prandtl-Blasius boundary-layer theory. Measurements of $R e_{\text {eff }}$ for the classical RBC regime in the RBC sample used for the present work gave $R e_{\text {eff }} \propto R a^{\zeta}$ with $\zeta=0.423 \pm 0.01$ (He et al. 2012b). This result is consistent with the model by Grossmann \& Lohse (2001) for a Reynolds number based on the LSC mean-flow velocity. Unfortunately, the available results for $\lambda_{u} / \lambda_{\text {th }}$ are inconsistent. The DNS results of Stevens et al. (2011) (see their figure 6b) and the measurements by du Puits, Resagk \& Thess (2009) (see their table 1), both for $\operatorname{Pr}=$ 0.7 and for $R a$ up to approximately $10^{12}$, suggest a ratio that is independent of $R a$. The same conclusion follows from Prandtl-Blasius boundary-layer theory (Shishkina et al. 2010). This leads to $T_{*} / \Delta T \propto R e_{s}^{-1 / 2} \propto R a^{-\eta_{t h}}$ with $\eta_{t h}=0.106 \pm 0.003$ (where the cited uncertainty assumes that $\lambda_{u} / \lambda_{t h}$ is independent of $R a$ ), which agrees fairly well with the experimental value $\eta=0.123 \pm 0.003$ derived above from the data shown in figure 7. On the other hand, the model of Grossmann \& Lohse (GL) with coefficients from Stevens et al. (2013) gives $\lambda_{u} / \lambda_{t h} \propto R a^{0.10}$. This result yields the theoretical exponent $\eta_{t h}=0.005$, which is smaller than the experimental value.

For completeness, we also consider the value $\operatorname{Pr}=12.3$ of Wei \& Ahlers (2014). For that case, the Reynolds number and $\lambda_{u} / \lambda_{\text {th }}$ from the GL model (see Stevens 


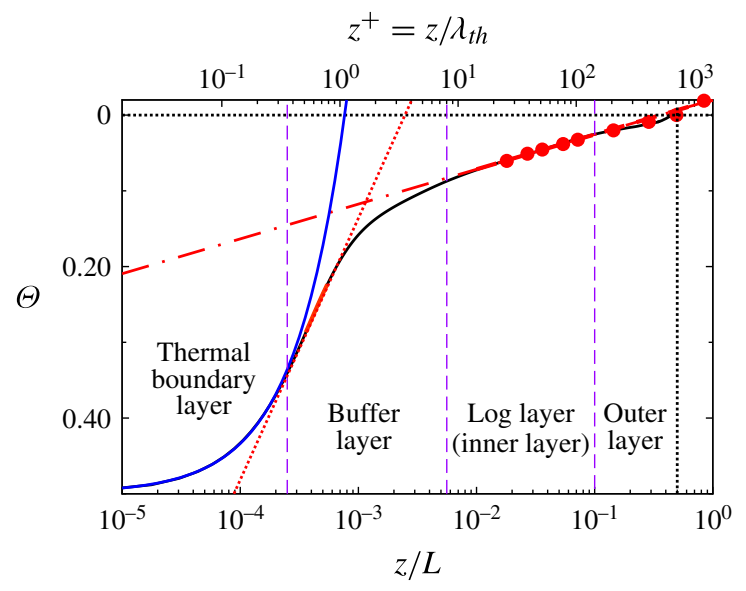

FIgURE 9. (Colour online) The dimensionless temperature $\Theta$ as a function of $z / L$ or $z / \lambda_{\text {th }}$ for $R a=2 \times 10^{12}$ and $\xi=0.018$. Solid circles (red online): run 1209041 (the same data as those shown in figures 3 and 4). Dash-dotted line (red online): fit of (1.2) to the data for $z / L<0.08$. Solid black line: DNS for $R a=2 \times 10^{12}$ from figure 3 of Ahlers et al. $(2012 a)$. Short-dashed line (blue online): fit of $\Theta=\Theta_{0} *(z / L)$ to the DNS data for $z / L \leqslant 10^{-4}$, which gave $\Theta_{0}=-670$. Dotted line (red online): fit of (1.2) to the DNS data over the narrow range indicated by the thick solid section (red). The vertical longer-dashed lines (purple online) show the approximate locations of the boundaries between four different regions, in analogy to those for the velocity in shear flow.

et al. 2013, and references therein) give $\zeta=0.44$ and $\lambda_{u} / \lambda_{t h} \propto R a^{0.10}$. These results yield $\eta_{t h}=0.01$, which corresponds to a weaker dependence of $A$ on $R a$ than was found for $\operatorname{Pr}=0.8$. This result is consistent with the measurements by Wei \& Ahlers (2014), in which the logarithmic temperature profile in a fluid with $\operatorname{Pr}=12.3$ revealed an $R a$-independent $A$ within the resolution of the measurement. However, the DNS of Shishkina, Wagner \& Horn (2014), as well as the Prandtl-Blasius analysis of Shishkina et al. (2010), yield an $R a$-independent $\lambda_{u} / \lambda_{\text {th }}$ over a wide range of $P r$, which in turn will give $\eta_{t h}=0.10$, in disagreement with the experimental value.

\subsubsection{Comparison between the profiles in turbulent convection and shear flow}

As (4.8) shows, the near-wall temperature is specified by $T_{*}$, which is similar to $u_{*}$ for the near-wall turbulence. Both $R a$-independent parameters, $\kappa_{\theta}$ and $f(P r)$, have their one-to-one correspondence with parameters of the equations for the velocity LoW. Therefore, the model discussed in $\S 4.3 .1$ represents close analogies between the timeaveraged temperature profiles in high-Rayleigh-number $\mathrm{RBC}$ and the time-averaged velocity profiles in high-Reynolds-number shear flows. Of course, there remains the important physical difference that in the shear-flow case the log profile is due to excitations ('coherent eddies') generated by the shear instability near the wall, while in the RBC case the thermal excitations ('plumes') that lead to the log profile in the temperature field are generated by the instability of the thermal boundary layer.

Using figure 9, we call attention to these close analogies. To this end, the scale of $\Theta$ is increasing in the downward direction, thus emphasizing the similarity to the shape of the downstream shear-flow velocity as a function of distance from the wall (see e.g. Pope 2000). The solid line in the figure is the result of DNS for $R a=2 \times 10^{12}$ and 
$\operatorname{Pr}=0.7$ (Stevens et al. 2011; as reported by Ahlers et al. 2012a). The solid circles are results of the present work.

We show $\Theta$ versus $z / L$ (bottom scale) and $z^{+} \equiv z / \lambda_{t h}$ (top scale). Here we use $\lambda_{t h}$ as the inner scale for calculating the distance from the plate in 'wall units' because $\lambda_{\text {th }} \equiv$ $L /(2 N u)$ can be directly calculated using the experimental data. With $N u=716$ from the present experiment, we find $\lambda_{t h} / L \simeq 7.0 \times 10^{-4}$ or $\lambda_{t h}=1.56 \mathrm{~mm}$ for the particular case shown here. The relation between $z^{+}$and the 'wall units' for the velocity profile $y^{+} \equiv z / \delta_{v}$ is given by $y^{+}=z^{+} \lambda_{t h} / \delta_{v}$. Using the observed $\lambda_{t h}=\lambda_{u}$ for $\operatorname{Pr} \simeq 0.8$ from the experimental and DNS data, one can estimate from (4.33) that $y^{+} \sim z^{+} R e_{s}^{1 / 2}$. For $R a=2 \times 10^{12}$, this estimation yields $y^{+}=O(10) z^{+}$.

Looking at figure 9, one sees that there is a thermal boundary layer for $z^{+} \lesssim 0.4$ where the temperature profile is well approximated by a linear function $\Theta \propto z^{+}$(the short dashed line). In the 'wall units' of $y^{+}$, this linear thermal sublayer extends to $y^{+} \sim 4$. We take it to play a role analogous to that of the 'viscous sublayer' in shear flow where the velocity varies linearly with $y^{+}$up to $y^{+} \simeq 5$ (see figure 7.8 in Pope (2000)).

Beyond the thermal BL, there is a 'buffer layer' that provides the crossover from the linear dependence of $\Theta$ on $z^{+}$to the logarithmic dependence. The buffer layer extends to $z^{+} \simeq 8$, which corresponds to an estimated value $y^{+} \simeq 80$. This is somewhat larger than the value of $y^{+} \simeq 30$ at the upper limit of the shear-flow buffer layer, and closer to the upper limit of the viscous wall region at $y^{+} \simeq 50$.

Over the range $8 \lesssim z^{+} \lesssim 150$, the log layer is found in the RBC system, corresponding to an estimated $\log$ layer $80 \lesssim y^{+} \lesssim 1500$. This estimated log region of $y^{+}$in the RBC temperature profile is consistent with the log-law region in shear flows within the order of magnitude (see e.g. Wei \& Wilmarth 1989; Pope 2000).

For $z^{+} \gtrsim 150$ (or $z / L \gtrsim 0.1$ ) there is an 'outer layer', which corresponds to the estimated region of $y^{+} \gtrsim 1500$. It is analogous to the outer layer in shear flow, where the log law does not hold. The experimental data show small deviations from the $\log$ profile in this region, which are barely noticeable in figure 9 but clearly seen in figure 4.

It is interesting to note that recent measurements of the spectra of fluctuations in this system (He et al. 2014) showed that the same analogy extends also to the dynamics. The data revealed that the fluctuation power varies with the frequency $f$ as $f^{-1}$ over about one decade of $f$ when $z$ is in the log-layer range of the variance of the fluctuating temperature. According to these analogies, we assume that thermal plumes in turbulent $\mathrm{RBC}$, which are the thermal excitations emitted from the BLs, play a role analogous to the velocity excitations, or 'coherent eddies', in shear flow (see e.g. Townsend 1976; Perry \& Chong 1982; Perry, Henbest \& Chong 1986). Similar to the predictions from the attached-eddy hypothesis by Townsend (1976), it is expected that the temperature variance follows the log law and that the temperature spectra scale as $f^{-1}$ in the log layer.

In contrast, an $f$ dependence with a more negative exponent, closer to $f^{-1.5}$, was found in an outer layer. This is not unlike the Kolmogorov scaling $f^{-1.7}$ found for frequency spectra in an outer layer near the centre of pipe flow (Rosenberg et al. 2013). Thus, we expect that the range $z^{+} \gtrsim 150$ does indeed correspond to an outer layer beyond the log-law region similar to that found in shear flows.

Finally, we note an important difference between shear flows and RBC. In fully developed turbulent shear flows, the system is homogeneous in the downstream ('streamwise') direction, with significant variations of the turbulent flow properties only in the transverse ( $y$, 'spanwise') direction. RBC is more complex because its 
(a)

$\Theta$

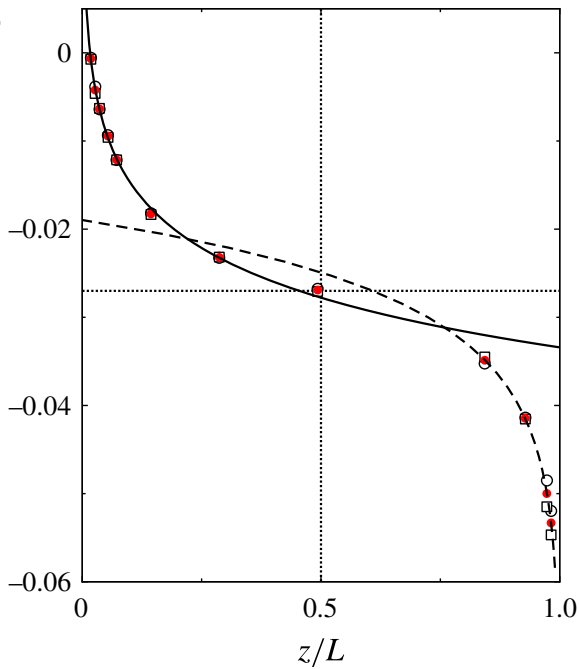

(b)

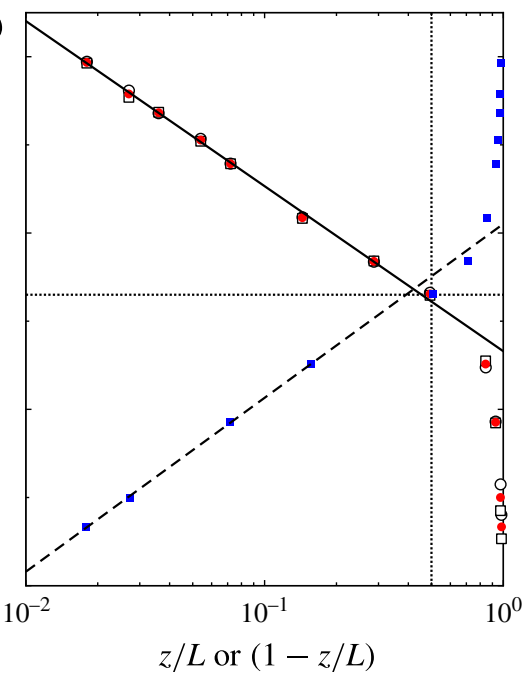

FIgURE 10. (Colour online) The dimensionless temperature $\Theta(z / L)$ as a function of $z / L$ or $(1-z / L)$ for $\xi=0.018, \Delta T=15.22 \mathrm{~K}, R a=9.94 \times 10^{14}$ and $P r=0.858$ (run 1207281 ). The open circles (squares) are from columns $\mathrm{V}_{0}\left(\mathrm{~V}_{1}\right)$ and $\mathrm{V}_{0}^{\prime}\left(\mathrm{V}_{1}^{\prime}\right)$. The solid circles (red online) and squares (blue online) are their average. (a) The data are shown on linear scales. (b) The same data are shown on a logarithmic horizontal scale. The solid and dashed lines are fits of (1.2) and (1.3) to the data for $z / L<0.08$ and $1-z / L<0.08$, respectively. The vertical dotted line is located at $z / L=1 / 2$ and the horizontal dotted line passes through the measured value of $\phi=\Theta(z / L=0.5)=-0.0270$. For this run the Nusselt number was 5540 .

properties vary in two dimensions, namely in the vertical $(z)$ direction (corresponding to the transverse shear-flow direction) and in the horizontal $(r)$ direction, which has no analogue in shear flow. Thus, as we saw in $\S 4.1 .5$, the parameters $A(r)$ and $B(r)$ of the logarithmic profiles are not constant in the horizontal plane but rather (in our cylindrical geometry) depend on the radial position. This is consistent with the experimental observation, as well as with findings from DNS, that plumes tend to rise (fall) mostly in up-flow (down-flow) regions of the LSC, with an associated plume density that is dependent on the radial position within the sample.

\section{Results for the ultimate state}

\subsection{Results for $R a=9.9 \times 10^{14}$}

\subsubsection{Axial dependence of $\Theta$ : the logarithmic temperature profile}

In figure 10 we show the reduced temperature $\Theta(z, r)$ (see (1.1)) at a radial position $\xi=0.0180$ as a function of $z / L$. As in figure 3, the points for $z / L \leqslant 0.5$ are from columns $\mathrm{V}_{0}$ and $\mathrm{V}_{1}$, and the points for $z / L>0.5$ are from $\mathrm{V}_{0}^{\prime}$ and $\mathrm{V}_{1}^{\prime}$. For these data $R a=9.94 \times 10^{14}$ (i.e. the sample is in the ultimate state) and $\operatorname{Pr}=0.858(P=$ 17.69 bar, $T_{m}=21.47^{\circ} \mathrm{C}, \Delta T=15.22 \mathrm{~K}$ ). As seen and discussed already for the classical state, the data from $\mathrm{V}_{0}$ and $\mathrm{V}_{0}^{\prime}\left(\mathrm{V}_{1}\right.$ and $\left.\mathrm{V}_{1}^{\prime}\right)$ differ slightly from each other, and we shall use their averages for further analysis.

From figure $10(b)$ it is clear that the data are described well by (1.2) and (1.3). The solid and dashed lines in panels $(a)$ and $(b)$ are fits of these equations to the solid circles or squares. For all fits in this paper, we used only the five data points 


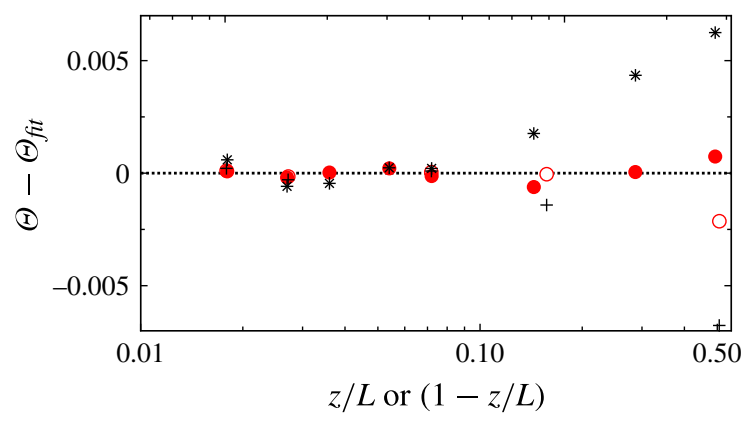

FIGURE 11. (Colour online) Deviations of the solid circles in figure 10 from a fit of (1.2) to the five points for $z / L<0.08$ (solid circles, red online) or of (1.3) to the three points for $(1-z / L)<0.08$ (open circles, red online). Note that this figure is rendered on the same scale as figure 4 to facilitate easy comparison. Also for easy comparison we show the points from figure 4 as black stars and plus signs.

with $z / L<0.08$ near the bottom plate and the three points with $(1-z / L)<0.08$ near the top plate. The fits gave $A=-0.00813 \pm 0.00017, B=-0.0334 \pm 0.0006$, $A^{\prime}=0.00857$ and $B^{\prime}=-0.0190$ (meaningful uncertainties for $A^{\prime}$ and $B^{\prime}$ cannot be established because there are not enough data points). For the amplitude ratio, the data yield $-A / A^{\prime}=0.95$. Although we cannot reliably estimate the probable error of this ratio, we shall see below in figure $15(a)$ that the standard deviation of many similar measurements from their mean value is 0.012 . Thus we believe that there is a significant deviation from the perfectly symmetric case $-A=A^{\prime}$.

In figure 11 we show the deviations of $\Theta$ from the above fits as solid and open circles. Except for very close to the horizontal midplane $(z / L=(1-z / L)=0.5)$, the points generally fall within a fraction of a part per thousand of the fit, with a standard deviation of $2 \times 10^{-4}$ corresponding to $3 \mathrm{mK}$. This differs from what was found for the classical state, where deviations became apparent already for $z / L \gtrsim 0.1$ and $(1-$ $z / L) \gtrsim 0.1$ (for comparison the classical-state data from figure 4 are shown in figure 11 as stars and as plus signs). Thus, in the ultimate state, we find no real evidence for an outer layer, and the log layers seems to extend nearly to the sample centre. Consistent with this, the extrapolations of the two $\log$ profiles cross the horizontal line $\Theta=\phi$ when $Z_{0}=0.46$ and $Z_{0}^{\prime}=0.39$, much closer to the sample centre at $z / L=0.50$ than was the case for the classical state.

The near absence of an 'outer layer', at least for $z / L<0.5$, is consistent with the arguments by Grossmann \& Lohse (2011) for the ultimate state, which predict logarithmic profiles extending throughout the sample from two very thin thermal sublayers, one each near the sample top and bottom. In the OB case, the log profiles are expected to meet in the sample centre without any outer layer at $z / L=0.5$. It was suggested by S. Grossmann (2012, private communication) that the small deviation from the $\log$ profile, primarily for $z / L>0.5$, may be associated with non-OB effects (which caused a deviation of the amplitude ratio $A / A^{\prime}$ from unity) and the requirement that the temperature be an analytic function of $z / L$ throughout the sample.

\subsubsection{Radial dependence of $\Theta$}

In figure 12 we show results for $\Theta(z / L)$ at four different radial positions $\xi=(R-$ $r) / R$ for $R a=9.94 \times 10^{14}$. As for the classical state illustrated by figure 5 , one sees that the amplitude $A$ of the logarithm (i.e. the slope of the lines fitted to the data) 


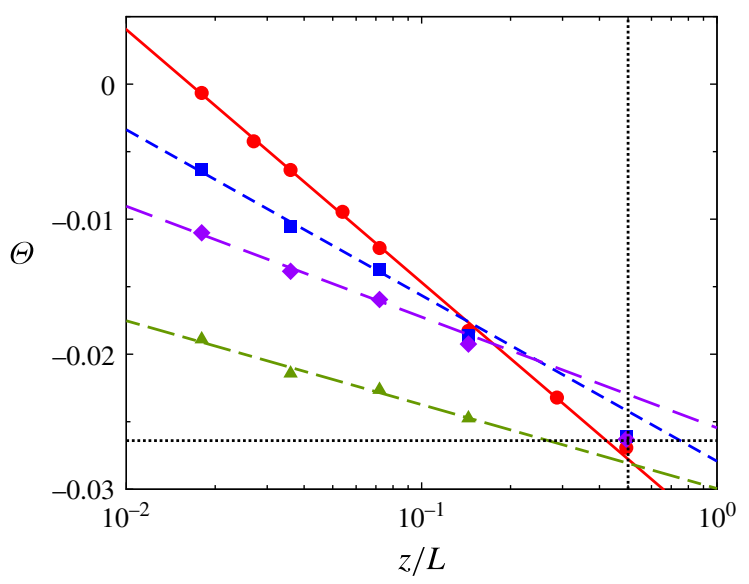

FIGURE 12. (Colour online) Results at the four radial locations for $\Theta(z, r)$ from run $1207281\left(R a=9.94 \times 10^{14}, P r=0.858\right)$. Near the left edge of the graph we have, from top to bottom, $\xi=(R-r) / R=0.0180,0.0356,0.0716$ and 0.2680 .

decreases as the radial position moves away from the sidewall into the sample interior. Remarkably, all four datasets yield the same value $\phi=\Theta(1 / 2)=-0.0267 \pm 0.0004$. Thus, as in the classical state, the measurements reveal no measurable deviation from a constant for the temperature in the horizontal midplane of the sample.

Figure 13 shows the absolute value $|A|$ of the amplitude of the logarithmic term (solid circles) as a function of $\xi$ on double-logarithmic scales. Also shown for comparison are the results for the classical state at $R a=2.0 \times 10^{12}$ from figure 6 (open circles). One sees that both datasets are described quite well by power laws, with very similar exponents. We also show the Grossmann \& Lohse (2012) prediction (4.3) for aspect ratio $\Gamma=1$, with $A_{1}=0.0035$ (dash-dotted line, red online) as shown already in figure 6 , and with $A_{1}=0.0012$ (dashed line, blue online), which passes through the ultimate state data. One sees that in both cases the fit is not very good; but as pointed out by Grossmann \& Lohse (2012), in their model the more nearly elliptic path of any LSC in a sample with $\Gamma=0.5$ would tend to produce a more rapid radial variation of $|A|$.

\subsection{Dependence of the parameters on $R a$}

In the ultimate state it is difficult to establish the $R a$ dependence of any parameter with meaningful accuracy because the amplitudes of the logarithmic term are smaller than in the classical state, and because the data cover at best a factor of 2 in $R a$. Nonetheless, the results for the amplitude $A$ of the logarithmic term given in figure 14 indicate that the magnitude of $A$ decreases as $R a$ increases at all three radial positions shown. This is similar to the $R a$ dependence found for the classical state.

For the radial position $\xi=0.0180$, an extra effort was made to obtain the best possible statistics by completing two measurement sequences, one starting with run 1207231 (circles) and the other with run 1209171 (triangles). There is no significant difference between the results. A fit of (4.4) to all those data (solid circles and triangles) yielded

$$
A_{0}=-4.8 \pm 3.0, \quad \eta=0.185 \pm 0.018 .
$$




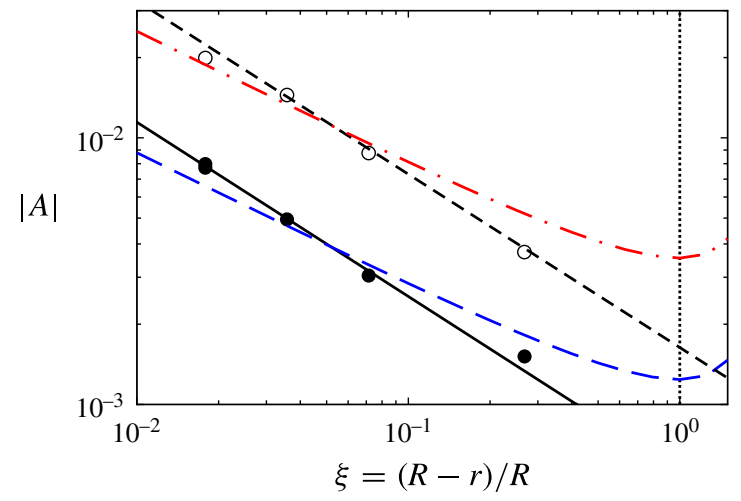

FIGURE 13. (Colour online) The absolute value $|A|$ of the amplitude of the logarithmic temperature profiles in figures 6 and 12. Open circles: $R a=2.0 \times 10^{12}$, classical state. Solid circles: $R a=1.049 \times 10^{15}$, ultimate state. Short dashed line: $|A|=0.0016 \xi^{-0.65}$. Solid line: $|A|=0.00056 \xi^{-0.65}$. Dash-dotted line (red online): (4.3) with $A_{1}=0.00354$. Long dashed line (blue online): (4.3) with $A_{1}=0.00124$. The vertical dotted line is located at the axis of the sample.

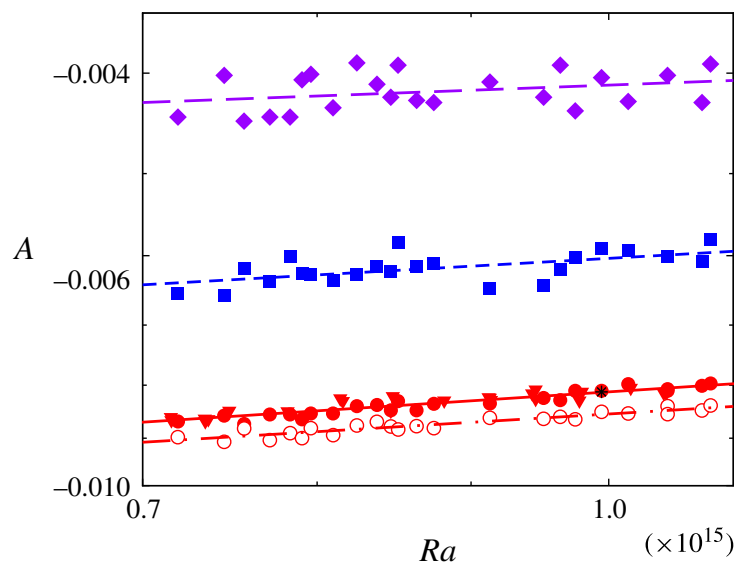

FIgURE 14. (Colour online) The amplitude $A$ of the logarithmic dependence of $\Theta$ on $z / L$ for the ultimate state as a function of the Rayleigh number $R a$. Circles and downwardpointing triangles (red online): $\xi=0.0180$. Squares (blue online): $\xi=0.0356$. Diamonds (purple online): $\xi=0.0716$. Solid symbols: $A$ from fits to data with $z / L<0.08$. Open circles: $-A^{\prime}$ from fits to data with $1-z / L<0.08$. The black star corresponds to the data in figure 10.

A fit of (4.5) to the open circles, for the same $\xi$ but for $(1-z / L)<0.08$, gave

$$
A_{0}^{\prime}=-3.7 \pm 2.1, \quad \eta^{\prime}=0.176 \pm 0.017 \text {. }
$$

At the other radial positions, the scatter is large and it is not possible to determine $\eta$ with good accuracy. The power-law fits yield $\eta=0.18 \pm 0.05$ for $\xi=0.0356$ (squares) and $\eta=0.14 \pm 0.13$ for $\xi=0.0716$ (diamonds).

We conclude that the above analysis does not reveal any radial dependence of $\eta$, and for $\xi=0.0180$ (where the accuracy is best) all the data are described well by 
(a)

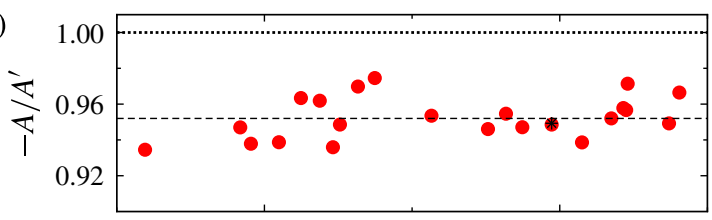

(b)

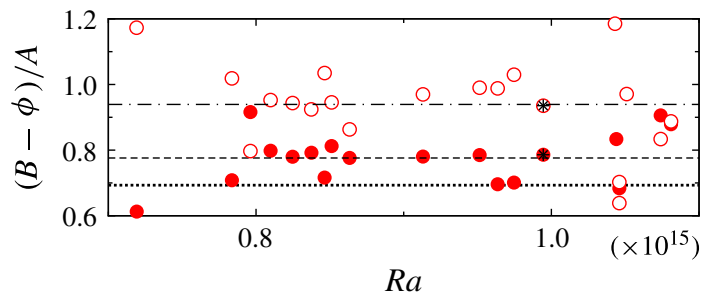

FIgURE 15. (Colour online) (a) The parameter ratio $-A / A^{\prime}$ and $(b)$ the ratios $(B-\phi) / A$ (solid circles) and $\left(B^{\prime}-\phi\right) / A^{\prime}$ (open circles) of the logarithmic dependence of $\Theta$ on $z / L$ for the ultimate state as a function of the Rayleigh number $R a$. In panel $(a)$ the short dashed line corresponds to the mean value $A / A^{\prime}=0.952 \pm 0.012$. In panel $(b)$ the dotted horizontal line corresponds to $(B-\phi) / A=-\ln (1 / 2) \simeq 0.693$, and the short-dashed and dash-dotted lines correspond to the average values $\langle(B-\phi) / A\rangle=0.776$ and $\left\langle\left(B^{\prime}-\phi\right) / A^{\prime}\right\rangle=$ 0.94 , respectively. The results for the run shown in figure 10 are indicated by the black stars.

$\eta=\eta^{\prime}=0.18 \pm 0.02$. This exponent value differs by nearly three standard deviations from the result (4.6) and (4.7) for the classical state. Thus we regard it as likely that the exponents of the power-law representations of $A(R a)$ differ in the two states. When $\eta=\eta^{\prime}$ is fixed at 0.18 , we find $A_{0}=-4.08 \pm 0.01$ and $A_{0}^{\prime}=4.27 \pm 0.02$, giving $A_{0} / A_{0}^{\prime}=$ $-0.955 \pm 0.006$. Thus the difference between the amplitudes $A$ and $A^{\prime}$ is significant. This differs from the result $A / A^{\prime}=-0.988 \pm 0.005$ for the classical state (see $\S 4.2$ ), which did not rigorously rule out the symmetric case $A=-A^{\prime}$.

One can also examine the amplitude ratio for individual $R a$ values. This is done in figure 15(a). The data reveal no $R a$ dependence for $-A / A^{\prime}$. The average of the data in the figure is 0.952 , with a standard deviation of 0.012 . This result is consistent with that cited in the previous paragraph, and both analyses indicates that the OB reflection symmetry about the horizontal midplane is broken.

In figure $15(b)$ we show $\left(B^{\prime}-\phi\right) / A^{\prime}$ (open circles) and $(B-\phi) / A$ (solid circles) as a function of $R a$. Although there is considerable scatter, the data do not reveal any dependence on $R a$ and give the average values $\left\langle\left(B^{\prime}-\phi\right) / A^{\prime}\right\rangle=0.939$ corresponding to $Z_{0}^{\prime}=0.39$ and $\langle(B-\phi) / A\rangle=0.776$ corresponding to $Z_{0}=0.46$. Thus we see that the log layer in the lower half of the sample extends essentially to the horizontal midplane $\left(Z_{0}=1 / 2\right)$, while in the upper half it extends less far and space remains for an outer layer. The value $Z_{0}^{\prime}=0.39$ is similar to the result $Z_{0}^{\prime}=0.36 \pm 0.01$ found in the classical state (see $\S 4.1 .4$ ). Thus, also this parameter indicates a lack of reflection symmetry about the midplane for the ultimate state.

\section{Summary}

In this paper we have presented detailed measurements of the logarithmic temperature profiles for both the classical and the ultimate state of turbulent $\mathrm{RBC}$ over the ranges $6 \times 10^{11} \lesssim R a \lesssim 5 \times 10^{12}$ and $7 \times 10^{14} \lesssim R a \lesssim 1.1 \times 10^{15}$, respectively. 
Even though non-OB effects shifted the centre temperature slightly away from the mean temperature, we found for the classical state that the amplitudes $A$ and $A^{\prime}$ are equal within our resolution. We show that deviations from the logarithmic profile occur for $z / L \gtrsim 0.1$ and $1-z / L \gtrsim 0.1$, indicating the existence of an outer layer beyond the $\log$ layer. The widths of the $\log$ and the outer layers are (within our resolution) independent of $R a$ and symmetric about the horizontal midplane. We discuss the radial dependence of $A$ and $A^{\prime}$ in terms of a power-law dependence, which leads to an effective exponent of approximately 0.65 , and in terms of a functional form proposed by Grossmann \& Lohse (2012) for the ultimate state. We note that the radial dependences of $A$ and $A^{\prime}$ presumably are a characteristic of samples with aspect ratios of order unity, which would not prevail in a laterally extended system, where the diffusion in the horizontal plane of many LSC cells would provide lateral averaging.

For $R a=2 \times 10^{12}$ the agreement of DNS with the measurements at the same $R a$ is remarkably good at radial positions not too near the centreline.

In $\S 4.2$ we presented the dependence of $A$ on $R a$ for the classical state. There we found that $A(R a, \xi=0.018)=A_{0} R a^{-\eta}$ with $\eta \simeq 0.123 \pm 0.03$ (at other $\xi$ the data were not sufficiently reliable to determine $\eta$ with meaningful accuracy). We note that very recent measurements for a much larger $\operatorname{Pr}=12.3$ (Wei \& Ahlers 2014) yielded an $R a$-independent $A$ and $A^{\prime}$, which suggests that $\eta$ is dependent upon $\operatorname{Pr}$.

In $\S 4.3$ we concluded the discussion of the classical state with an outline of the analogies, similarities and differences between RBC and shear flows. First, in $\$ 4.3 .1$ we present a derivation of the logarithmic profiles from a two-layer model of the temperature profile that is similar to the model developed by Grossmann \& Lohse (2012) for the ultimate state. We find that $A \simeq R a^{-\eta_{t h}}$ with $\eta_{t h}=0.106$, in quite good agreement with the measurements. Then, in $\$ 4.3 .2$, we show that the domain diagram for RBC, with a thermal boundary layer, a buffer layer, a log (inner) layer and an outer layer, is very similar to the equivalent diagram for shear flows when, in the RBC case, length is scaled by the thermal BL thickness $\lambda_{t h}$.

In $\S 5$ we presented results for the ultimate state in the same sequence as had been done in $\S 4$ for the classical state. We shall not describe them in detail here because, quite remarkably, at a qualitative level, the observed phenomena are very similar to those found below $R a^{*}$, and even quantitatively there are few distinctions. However, some important differences do become apparent. Thus, within our resolution, the dependence of $A$ on $R a$ yields a slightly larger exponent $\eta \simeq 0.18$. This value is significantly larger than the effective exponent prediction $\eta_{G L} \simeq 0.043$ of Grossmann \& Lohse (2012) for the ultimate state. We find that the log layer in the lower half of the sample extends up to the horizontal midplane, as had been predicted by Grossmann \& Lohse (2012). In the upper half the $\log$ amplitude $A^{\prime}$ is slightly smaller than $A$, suggesting an influence of deviations from the OB approximation. Given the unequal $\log$ amplitudes, it is not possible for both log layers to meet in the sample centre because this would lead to a singularity in the temperature profile; thus, as originally suggested by S. Grossmann (2012, private communication), the required analyticity of $\Theta(z)$ may explain the deviations from the $\log$ profile in the upper half when $1-z / L \gtrsim$ 0.3 or so.

Finally, we note that previous measurements of vertical temperature profiles sometimes were fitted to logarithmic functions. For instance, the data of Zhou \& Xia (2013) along the centreline of a sample in the shape of a rectangular cuboid and with $\operatorname{Pr}=4.3, R a=O\left(10^{11}\right)$ and $0.002 \lesssim z / L \lesssim 0.02$ could be represented by (1.2). However, in our view, the range of $z / L$ overlaps the buffer layer (see figure 9 ) 
and the values derived for the amplitudes $|A|$ of the logarithm are too large to be representative of the log layer ('inner layer', see figure 9). They are an order of magnitude larger than those obtained recently along the centreline of a cylindrical sample with $\operatorname{Pr}=12.3$ (Wei \& Ahlers 2014). Another range of $z$ over which a logarithmic dependence has sometimes been thought to fit the data is near the crossover from the thermal boundary layer to the buffer layer (see figure 9). There, on the semi-logarithmic representation of $\Theta$, a straight line can be drawn through the DNS results over a small range of $z$ (see the dotted red line in the figure). This, however, is illusory and merely a consequence of the existence of a point of inflection of the curve. The issue was examined in some detail by Shishkina \& Thess (2009), who demonstrated explicitly that a logarithmic function is not appropriate for this range of $z$.

\section{Acknowledgements}

We thank A. Kopp, A. Kubitzek and A. Renner for their enthusiastic technical support. We are very grateful to $\mathrm{H}$. Nobach for many useful discussions and for his contributions to the assembly of the experiment. We benefited from numerous discussions with S. Grossmann and D. Lohse. We are very grateful to the Max-Planck-Society and the Volkswagen Stiftung, whose generous support made the establishment of the facility and the experiments possible. We thank the Deutsche Forschungsgemeinschaft (DFG) for financial support through SFB963: 'Astrophysical Flow Instabilities and Turbulence'. The work of G.A. was supported in part by the US National Science Foundation through grant DMR11-58514.

\section{REFERENCES}

Ahlers, G. 2009 Turbulent convection. Physics 2, 74.

Ahlers, G., Bodenschatz, E., Funfschilling, D., Grossmann, S., He, X., Lohse, D., SteVens, R. \& Verzicco, R. $2012 a$ Logarithmic temperature profiles in turbulent RayleighBénard convection. Phys. Rev. Lett. 109, 114501.

Ahlers, G., Brown, E., Fontenele Araujo, F., Funfschilling, D., Grossmann, S. \& LoHSE, D. 2006 Non-Oberbeck-Boussinesq effects in strongly turbulent Rayleigh-Bénard convection. J. Fluid Mech. 569, 409-445.

Ahlers, G., Calzavarini, E., Fontenele Araujo, F., Funfschilling, D., Grossmann, S., Lohse, D. \& SugiYama, K. 2008 Non-Oberbeck-Boussinesq effects in turbulent thermal convection in ethane close to the critical point. Phys. Rev. E 77, 046302.

Ahlers, G., Fontenele Araujo, F., Funfschilling, D., Grossmann, S. \& Lohse, D. 2007 Non-Oberbeck-Boussinesq effects in gaseous Rayleigh-Bénard convection. Phys. Rev. Lett. 98, 054501.

Ahlers, G., Funfschilling, D. \& Bodenschatz, E. $2009 a$ Transitions in heat transport by turbulent convection for $\operatorname{Pr}=0.8$ and $10^{11} \leqslant R a \leqslant 10^{15}$. New J. Phys. 11, 123001.

Ahlers, G., Grossmann, S. \& Lohse, D. $2009 b$ Heat transfer and large scale dynamics in turbulent Rayleigh-Bénard convection. Rev. Mod. Phys. 81, 503-538.

Ahlers, G., He, X., Funfschilling, D. \& Bodenschatz, E. $2012 b$ Heat transport by turbulent Rayleigh-Bénard convection for $\operatorname{Pr} \simeq 0.8$ and $3 \times 10^{12} \lesssim R a \lesssim 10^{15}$ : aspect ratio $\gamma=0.50$. New J. Phys. 14, 103012.

Bailon-Cuba, J., Emran, M. S. \& Schumacher, J. 2010 Aspect ratio dependence of heat transfer and large-scale flow in turbulent convection. J. Fluid Mech. 655, 152-173.

BoussinesQ, J. 1903 Theorie Analytique de la Chaleur, vol. 2. Gauthier-Villars.

Brown, E. \& Ahlers, G. 2007 Temperature gradients, and search for non-Boussinesq effects, in the interior of turbulent Rayleigh-Bénard convection. Europhys. Lett. 80, 14001. 
Chavanne, X., Chillà, F., Castaing, B., Hébral, B., Chabaud, B. \& Chaussy, J. 1997 Observation of the ultimate regime in Rayleigh-Bénard convection. Phys. Rev. Lett. 79, 3648-3651.

Chavanne, X., Chillà, F., Chabaud, B., Castaing, B. \& Hébral, B. 2001 Turbulent RayleighBénard convection in gaseous and liquid He. Phys. Fluids 13, 1300-1320.

Chillà, F. \& Schumacher, J. 2012 New perspectives in turbulent Rayleigh-Bénard convection. Eur. Phys. J. E 35, 58.

Churchill, S. W. 2002 A reinterpretation of the turbulent Prandtl number. Ind. Engng Chem. Res. 41, 6393-6401.

Grossmann, S. \& Lohse, D. 2000 Scaling in thermal convection: a unifying view. J. Fluid Mech. 407, 27-56.

Grossmann, S. \& Lohse, D. 2001 Thermal convection for large Prandtl number. Phys. Rev. Lett. 86, 3316-3319.

Grossmann, S. \& Lohse, D. 2002 Prandtl and Rayleigh number dependence of the Reynolds number in turbulent thermal convection. Phys. Rev. E 66, 016305.

Grossmann, S. \& LohSE, D. 2011 Multiple scaling in the ultimate regime of thermal convection. Phys. Fluids 23, 045108.

Grossmann, S. \& Lohse, D. 2012 Logarithmic temperature profiles in the ultimate regime of thermal convection. Phys. Fluids 24, 125103.

He, X., Funfschilling, D., Bodenschatz, E. \& Ahlers, G. $2012 a$ Heat transport by turbulent Rayleigh-Bénard convection for $\operatorname{Pr} \simeq 0.8$ and $4 \times 10^{11} \lesssim R a \lesssim 2 \times 10^{14}$ : ultimate-state transition for aspect ratio $\gamma=1.00$. New J. Phys. 14, 063030.

He, X., Funfschilling, D., Nobach, H., Bodenschatz, E. \& Ahlers, G. $2012 b$ Transition to the ultimate state of turbulent Rayleigh-Bénard convection. Phys. Rev. Lett. 108, 024502.

He, X., Funfschilling, D., Nobach, H., Bodenschatz, E. \& Ahlers, G. 2013 Comment on 'Effect of boundary layers asymmetry on heat transfer efficiency in turbulent Rayleigh-Bénard convection at very high Rayleigh numbers' by Urban et al. Phys. Rev. Lett. 110, 199401.

He, X., van Gils, D., Bodenschatz, E. \& Ahlers, G. 2014 Logarithmic spatial variations and universal $f^{-1}$ power spectra of temperature fluctuations in turbulent Rayleigh-Bénard convection. Phys. Rev. Lett. 112, 174501.

HogG, J. \& AHLERS, G. 2013 Reynolds-number measurements for low-Prandtl-number turbulent convection of large aspect-ratio samples. J. Fluid Mech. 725, 664-680.

JisChA, M. \& RiEKE, H. B. 1979 About the prediction of turbulent Prandtl and Schmidt numbers from modeled transport equations. Intl J. Heat Mass Transfer 22, 1547-1555.

Kadanoff, L. P. 2001 Turbulent heat flow: structures and scaling. Phys. Today 54 (8), 34-39.

KADER, B. A. 1981 Temperature and concentration profiles in fully turbulent boundary layers. Intl J. Heat Mass Transfer 24, 1541-1544.

KADER, B. A. \& YAGlom, A. M. 1972 Heat and mass transfer laws for fully turbulent wall flows. Intl J. Heat Mass Transfer 15, 2329-2351.

VON KÁRMÁN, T. 1930 Mechanische ähnlichkeit und Turbulenz. Nachr. Ges. Wiss. Göttingen, Math.Phys. Kl. 58-76, 322-336.

Kraichnan, R. H. 1962 Turbulent thermal convection at arbritrary Prandtl number. Phys. Fluids 5, 1374-1389.

LAndaU, L. D. \& Lifshitz, E. M. 1963 Fluid Mechanics (3rd impression). Pergamon Press.

Lohse, D. \& XIA, K.-Q. 2010 Small-scale properties of turbulent Rayleigh-Bénard convection. Annu. Rev. Fluid Mech. 42, 335-364.

LUI, S. L. \& XIA, K.-Q. 1998 Spatial structure of the thermal boundary layer in turbulent convection. Phys. Rev. E 57, 5494-5503.

Malkus, M. V. R. 1954 The heat transport and spectrum of thermal turbulence. Proc. R. Soc. Lond. A 225, 196-212.

Marusic, I., McKeon, B. J., Monkewitz, P. A., Nagib, H. M., Smits, A. J. \& Sreenivasan, K. R. 2010 Wall-bounded turbulent flows at high Reynolds numbers: recent advances and key issues. Phys. Fluids 22, 065103. 
Niemela, J. J., Skrbek, L., Sreenivasan, K. R. \& Donnelly, R. 2000 Turbulent convection at very high Rayleigh numbers. Nature 404, 837-840.

OBERBECK, A. 1879 Über die Wärmeleitung der Flüssigkeiten bei Berücksichtigung der Strömungen infolge von Temperaturdifferenzen. Ann. Phys. Chem. 7, 271-292.

Perry, A. E. \& Chong, M. S. 1982 On the mechanism of wall turbulence. J. Fluid Mech. 119, $173-217$.

Perry, A. E., Henbest, S. \& Chong, M. S. 1986 A theoretical and experimental study of wall turbulence. J. Fluid Mech. 165, 163-199.

van Der Poel, E. P., Mónico, R. O., Grossmann, S. \& Lohse, D. 2013 Logarithmic mean temperature profiles in Rayleigh-Bénard convection simulations. In Proceedings of the 14th European Turbulence Conference, Lyon, France.

Pope, S. B. 2000 Turbulent Flows. Cambridge University Press.

PrandTL, L. 1925 Bericht über Untersuchungen zur ausgebildeten Turbulenz. Z. Angew. Math. Mech. 5, 136-139.

Prandtl, L. 1932 Zur turbulenten Strömung in Rohren und längs Platten. Ergeb. Aerodyn. Versuch. Göttingen IV, 18.

Priestley, C. H. B. 1954 Convection from a large horizontal surface. Aust. J. Phys. 7, 176-201.

Priestley, C. H. B. 1959 Turbulent Transfer in the Lower Atmosphere. University of Chicago Press.

DU Puits, R., Resagk, C. \& Thess, A. 2009 Structure of viscous boundary layers in turbulent Rayleigh-Bénard convection. Phys. Rev. E 80, 036318.

QIU, X. L. \& Tong, P. 2001 Large scale velocity structures in turbulent thermal convection. Phys. Rev. E 64, 036304.

ReICHARDT, H. 1951 Die grundlagen des turbulent Warmeuberganges. Arch. Gesamte Warmetechnik 2, 129-142.

Roche, P.-E., Gauthier, F., Kaiser, R. \& Salort, J. 2010 On the triggering of the ultimate regime of convection. New J. Phys. 12, 085014.

Rosenberg, B. J., Hultmark, M., Vallikivi, M., Bailey, S. C. C. \& Smits, A. J. 2013 Turbulence spectra in smooth- and rough-wall pipe flow at extreme Reynolds numbers. J. Fluid Mech. 731, 46-63.

She, Z.-S., Chen, X., Chen, J., Zou, H.-Y., Bao, Y. \& Hussain, F. 2014 Prediction of temperature distribution in turbulent Rayleigh-Bénard convection. arXiv:1401.2138v1.

Shishinina, O., Stevens, R., Grossmann, S. \& Lohse, D. 2010 Boundary layer structure in turbulent thermal convection and its consequences for the required numerical resolution. New J. Phys. 12, 075022.

ShISHKINA, O.\& THESS, A. 2009 Mean temperature profiles in turbulent Rayleigh-Bénard convection of water. J. Fluid Mech. 633, 449-460.

Shishinina, O., WAGNer, S. \& Horn, S. 2014 Influence of the angle between the wind and the isothermal surfaces on the boundary layer structures in turbulent thermal convection. Phys. Rev. E 89, 033014.

Smits, A. J., McKeon, B. J. \& Marusic, I. 2011 High Reynolds number wall turbulence. Annu. Rev. Fluid Mech. 43, 353-375.

Spiegel, E. A. 1971 Convection in stars. Annu. Rev. Astron. Astrophys. 9, 323-352.

Stevens, R. J. A. M., Lohse, D. \& Verzicco, R. 2011 Prandtl and Rayleigh number dependence of heat transport in high Rayleigh number thermal convection. J. Fluid Mech. 688, 31-43.

Stevens, R. J. A. M., van der Poel, E. P., Grossmann, S. \& Lohse, D. 2013 The unifying theory of scaling in thermal convection: the updated prefactors. J. Fluid Mech. 730, 295-308.

Stevens, R. J. A. M., Zhou, Q., Grossmann, S., Verzicco, R., Xia, K.-Q. \& Lohse, D. 2012 Thermal boundary layer profiles in turbulent Rayleigh-Bénard convection in a cylindrical sample. Phys. Rev. E 85, 027301.

Tilgner, A., Belmonte, A. \& Libchaber, A. 1993 Temperature and velocity profiles of turbulence convection in water. Phys. Rev. E 47, R2253-R2256.

Townsend, A. A. 1976 The Structure of Turbulent Shear Flow. Cambridge University Press. 
Urban, P., Hanzelka, P., Kralik, T., Musilova, V., Srnka, A. \& Skrbek, L. 2012 Effect of boundary layers asymmetry on heat transfer efficiency in turbulent Rayleigh-Bénard convection at very high Rayleigh numbers. Phys. Rev. Lett. 109, 154301.

WeI, P. \& Ahlers, G. 2014 Logarithmic temperature profiles in the bulk of turbulent Rayleigh-Bénard convection for a Prandtl number of 12.3. J. Fluid Mech. doi:10.1017/jfm.2014.560.

Wei, T. \& Wilmarth, W. 1989 Reynolds-number effects on the structure of turbulent flows. J. Fluid Mech. 204, 57-95.

WU, X. Z. 1991 Along a road to developed turbulence: free thermal convection in low temperature He gas. PhD thesis, University of Chicago, IL. [The data of Wu were re-evaluated on the basis of new fluid properties by Niemela et al. (2001, private communication)].

Wu, X. Z. \& Libchaber, A. 1991 Non-Boussinesq effects in free thermal convection. Phys. Rev. A 43, 2833-2839.

YAGLOM, A. M. 1979 Similarity laws for constant-pressure and pressure-gradient turbulent wall flows. Annu. Rev. Fluid Mech. 11, 505-540.

Yu, B., Ozoe, H. \& Churchill, S. W. 2001 The characteristics of fully developed turbulent convection in a round tube. Chem. Engng Sci. 56, 1781-1800.

Zhang, J., Childress, S. \& Libchaber, A. 1997 Non-Boussinesq effect: thermal convection with broken symmetry. Phys. Fluids 9, 1034-1042.

Zhou, Q., Sugiyama, K., Stevens, R., Grossmann, S., Lohse, D. \& Xia, K.-Q. 2010 Prandtl-Blasius temperature and velocity boundary-layer profiles in turbulent Rayleigh-Bénard convection. J. Fluid Mech. 664, 297-312.

Zhou, Q. \& XIA, K.-Q. 2010 Measured instantaneous viscous boundary layer in turbulent RayleighBénard convection. Phys. Rev. Lett. 104, 104301.

Zhou, Q. \& XIA, K.-Q. 2013 Thermal boundary layer structure in turbulent Rayleigh-Bénard convection in a rectangular cell. J. Fluid Mech. 721, 199-224. 